\title{
Radiation Field From a Rectangular Source
}

\author{
J. H. Hubbell, R. L. Bach, and J. C. Lamkin
}

(January 12, 1960)

\begin{abstract}
Many radiation shielding problems involve calculations of the response of an isotropic detector to radiation of arbitrary angular distribution from uniform rectangular sources. In calculations of this type the family of integrals $\int_{S}\left(\cos \theta d S / r^{2}\right) P_{l}(\cos \theta)$ and the integral $\int_{S}\left(d S / r^{2}\right) \exp (-\mu t / \cos \theta)$ are frequently encountered, where $\theta$ is obliquity with respect to an axis perpendicular to the plane containing the rectangular radiant surface, $S, r$ is the distance from an element of source area, $d S$, to the detector, $\mu$ is the attenuation coefficient, and $t$ is the barrier thickness. Solutions of the first type of integral facilitate use of Legendre expansion representations of radiation directional distributions, and may also have application in other radiant surface studies, such as illumination and heat exchange engineering. The second integral relates to exponentially attenuated radiation from a plane isotropic rectangular source separated from the detector by a layer of material of thickness $t$. Formulas, expansions, and numerical results are presented.
\end{abstract}

\section{Introduction}

The penetration of radiation into structures has been the subject of theoretical $[1,2]^{2}$ and experimental [3] investigations as part of a general program at the National Bureau of Standards and other agencies. The theoretical investigations are usually performed in two stages: (a) penetration and (b) geometry. The penetration stage refers to generation of data relating to interaction of the radiation with barrier materials [4], and geometry refers here to the application of such data to specific configurations of source, barrier, and detector. This paper treats the geometrical stage of problems involving rectangular sources, e.g., ceilings of buildings or shelters. We draw to some extent from similar treatments in illumination [5 to 8] and heatexchange [9] engineering. The methods developed in this paper, in turn, have application to rectangular sources of light and heat as well as gamma radiation.

In the geometrical stage of many shielding problems, the following situation, generalized in figure 1, arises: one has a radiation detector and a surface $S$ through which radiation emerges. In the schematization of reference [1], the flow of radiation through each element of surface, $d S$, is independent of position on the surface, and no scattering of radiation occurs after emergence from surface $S$. The intensity and angular distribution, $g(\cos \theta)$, of radiation crossing the plane $S$ is assumed known from the penetration stage of the analysis. The quantity $g(\cos \theta)$, usually called the $f u x$ [4], is the radiation intensity per solid angle (e.g., photons per $\mathrm{cm}^{2}$ per second per steradian) traveling in the direction $\theta$ with respect to the $z$-axis (see fig. 1 ). If the detector has an isotropic response it will register a value $I_{s}$ proportional to an integral of $g(\cos \theta)$ over the total solid angle, $\Omega_{S}$, subtended by the surface $S$ from the detector:

$$
I_{S}=\int_{S} d \Omega_{S}(\theta) g(\cos \theta) .
$$

where

$$
d \Omega_{S}(\theta)=d S \cdot \cos \theta / r^{2}
$$

and $r$ is the distance from surface element $d S$ to the detector.

Introducing the quantity $\psi_{S}(\theta)$, which is the total range of azimuthal angle, $\varphi$, defined by the intersection of a $\theta$-cone with the surface $S$, as in figure 1 , we may write (1) in polar coordinates as

\footnotetext{
1 Work supported jointly by the Office of Civil and Defense Mobilization, the Navy Bureau of Yards and Docks, and the Defense Atomic Support Agency.

2 Figures in brackets indicate the literature references at the end of this paper.
} 


$$
\begin{aligned}
I_{S} & =\int_{-1}^{1} d(\cos \theta) g(\cos \theta) \int_{0}^{\psi_{S}(\theta)} d \varphi \\
& =\int_{-1}^{1} d(\cos \theta) g(\cos \theta) \psi_{S}(\theta) .
\end{aligned}
$$

As in the example in figure $1, \psi_{S}(\theta)$ may consist of more than one "piece" of azimuth, $\Delta_{i} \varphi_{S}(\theta)$, so that in the general case it is a summation over all such pieces:

$$
\psi_{S}(\theta)=\Delta_{1} \varphi_{S}(\theta)+\Delta_{2} \varphi_{S}(\theta)+\ldots .
$$

This quantity is identical with the "angular response function," $\psi(\theta)$, of reference [1] except for a factor $2 \pi .^{3}$

The surface $S$ may now be specified as a rectangle, as shown in figure 2 , with corners at $x$ and $y$ coordinates $(0,0),(w, 0),(w, l)$, and $(0, l)$. The detector is on the z-axis, hereafter referred to as the "corner position," at $z=h$. Since the solid angle integration indicated in eq (1) is dependent on relative rather than absolute distances we may reduce the number of parameters by dividing all dimensions and coordinates by $h$, i.e.,

$$
a=w / h, \quad b=l / h, \quad a=x / h, \quad \text { and } \beta=y / h .
$$

This representation of the relative geometry, rather than representation by horizontal and vertical "eccentricities" as in references [1] and [2], is used here in order that the interchangeability of length and width might be used as a partial check of the results.

Figure 3 shows the use of these scaled coordinates in describing a typical rectangular source problem. Radioactive material is uniformly distributed over the roof slab of an underground shelter, and the problem is to calculate the detector response to radiation transmitted by the rectangular ceiling, or rectangular portions of the ceiling, into the enclosure below.

${ }_{3}$ Berger and Lamkin [1] included in $\psi(\theta)$ a normalizing factor $1 / 2 \pi$, here omitted. Thus the quantity $\psi_{\mathbf{S}}(\theta)$ defined in $(3)$, above, is equivalent to their azimuthal range parameter $A_{\theta}$.

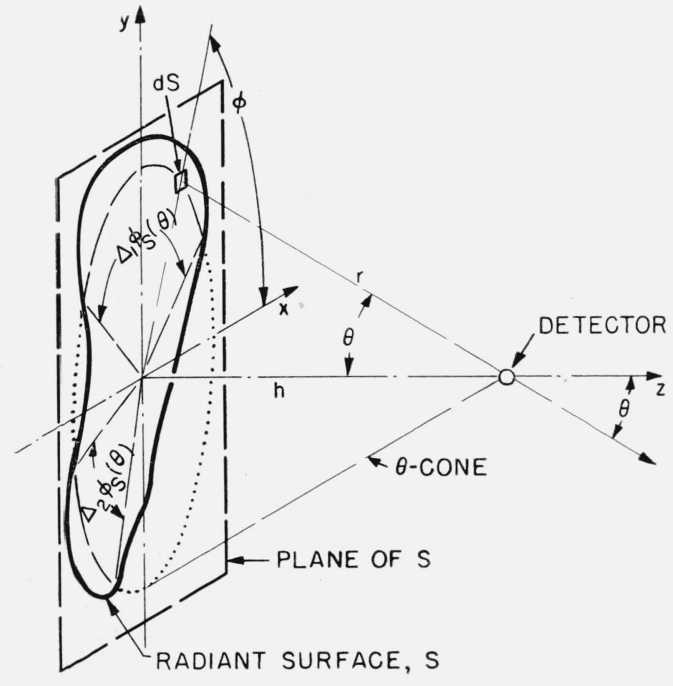

GURE 1. Geometrical schematization of a detector at distance $h$ from a plane source $S$ of arbitrary shape.

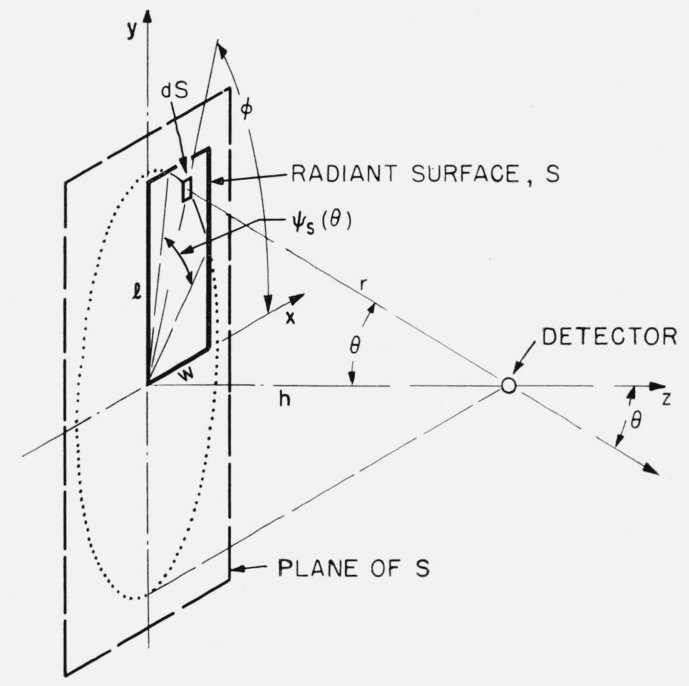

Figure 2. Schematization when $S$ is a rectangle of width $w$ and length $l$ with the detector in a "corner position" at distance $h$ from the plane of $S$. 


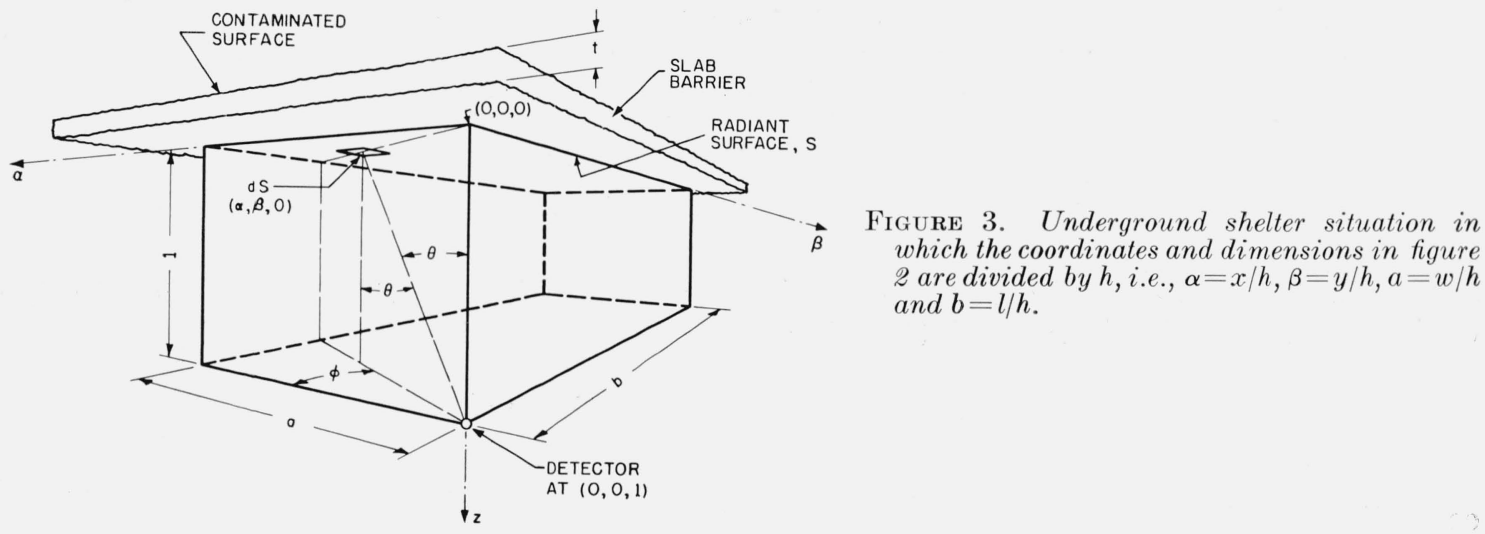

There are several ways to evaluate the integral (1) for a rectangle, either in polar form ( $2 \mathrm{a}, \mathrm{b})$ or using the cartesian parameters $\alpha, \beta$. Berger and Lamkin [1] performed the integrations numerically, using the polar form, for a few representative angular distributions $g(\cos \theta)$ and a variety of rectangular shapes. Spencer [2] has approximated the function $\psi_{S}(\theta)$, which may now be written for a rectangle as $\psi(\theta, a, b)$, by step functions so as to reduce $(2 \mathrm{~b})$ to a combination of values of the cumulative integral $\int_{x}^{1} d(\cos \theta) g(\cos \theta)$.

In this paper, advantage is taken of the fact that $g(\cos \theta)$ may usually be represented as a sum of Legendre polynomials, i.e.,

$$
g(\cos \theta)=\sum_{l=0}^{\infty}\left(l+\frac{1}{2}\right) g_{l} P_{l}(\cos \theta)
$$

where

$$
g_{l}=\int_{-1}^{1} d(\cos \theta) g(\cos \theta) P_{l}(\cos \theta) .
$$

Thus the integral (2b) may be expressed as a sum of elementary integrals

$$
I(a, b)=\sum_{l=0}^{\infty}\left(l+\frac{1}{2}\right) g_{l} p_{l}(a, b),
$$

where the $p_{l}(a, b)$ 's are coefficients of the expansion

$$
\psi(\theta, a, b)=\sum_{l=0}^{\infty}\left(l+\frac{1}{2}\right) p_{l}(a, b) P_{l}(\cos \theta) .
$$

The $p_{l}(a, b)$ 's, which are purely geometrical and independent of the penetration stage of the problem (i.e., source energy, barrier thickness and material, etc.), may be evaluated as

$$
p_{l}(a, b)=\int_{S} d \Omega_{S}(\theta) P_{l}(\cos \theta)
$$

in which $a$ and $b$ completely specify the surface $S$. Evaluation of the $\mathrm{p}_{l}(a, b)$ 's will be outlined in section 4.1 .

The sum (6) converges rapidly if either the $g_{l}$ or the $p_{l}$ converge rapidly. Since the $g_{l}$ 's are provided more readily from transport theory [4] than $g(\cos \theta)$, it is desirable to use (6) wherever applicable.

In the case of penetrating radiations, as from fallout, convergence of (6) may be inadequate as a result of the presence of the unscattered radiation component, which has an angular distribution of the form

$$
g^{o}(\tau, \cos \theta)=\frac{\sigma}{4 \pi} \exp (-\tau / \cos \theta) / \cos \theta .
$$


The superscript ${ }^{\circ}$ refers to unscattered radiation, $\tau$ is the slab thickness, $\mu t$, in mean-free-paths, and $\sigma$ is the surface density of radioactive material. Since the $g_{l}{ }^{o}(\tau)$ 's evaluated from (5) and (9) converge too poorly for use in the expansion (6) when $0 \leq \tau \lesssim 1$, an alternative expansion

$$
I^{o}(\tau, a, b)=\frac{\sigma}{4 \pi} \sum_{n=0}^{\infty} \tau^{n} q_{n}(a, b)
$$

is useful. The geometry-dependent coefficients $q_{n}(a, b)$, which are obtained by substituting (9) into (1), expanding in $\tau^{n}$ and integrating term by term, are given in section 5.2.

\section{Notation and Definitions}

Notation relevant to this paper, much of which has already been introduced, is listed for convenience as follows:

detector $=$ device with response proportional to the radiation flux at a given point in space,

$S=$ symbolic reference to a specific surface lying in the $x, y$ plane,

$h=$ distance from surface to detector in the $z$-direction,

$w, l=$ width and length of a rectangular surface,

$x, y=$ rectangular coordinates parallel to $w$ and $l$, respectively, with origin at one corner of the rectangle,

corner position $=$ detector position on a line perpendicular to the rectangle at one of its corners, i.e., at $(0,0, h)$,

$$
\begin{aligned}
& a=w / h, b=l / h, \\
& \alpha=x / h, \beta=y / h,
\end{aligned}
$$

$d S=$ element of area of the surface $S$,

$\theta=$ obliquity with respect to the $z$ axis,

$\varphi=$ azimuth of the plane through the $z$ axis and the center of $d S$, measured from the $x, z$ plane,

$\psi_{S}(\theta)=$ range of $\varphi$ covered by all surface elements $d S$ from which the detector can be seen at obliquity $\theta$,

$\psi(\theta, a, b)=\psi_{S}(\theta)$ when $S$ refers to a rectangle and the detector is in a corner position,

$r=$ distance from $d S$ to the detector,

$d \Omega_{S}(\theta)=$ solid angle subtended from the detector by element $d S$ at obliquity $\theta$

$=d S \cdot \cos \theta / r^{2}$,

$g(\cos \theta)=$ radiation flux (e.g., number of photons) traveling in direction $\theta$ per unit solid angle, unit time, and unit area normal to direction $\theta$,

$g_{l}=$ coefficients of a Legendre polynomial expansion of $g(\cos \theta)$,

$p_{l}=$ coefficients of a Legendre polynomial expansion of $\psi_{S}(\theta)$,

$I_{S}=$ response of an isotropic detector to surface $S$ radiating according to $g(\cos \theta)$, above,

$I(a, b)=I_{S}$ when $S$ is a rectangle and the detector is in a corner position,

$I(a, b ; \alpha, \beta)=I_{S}$ when the detector lies at $(h \alpha, h \beta, h)$.

The remaining quantities refer specifically to applications involving penetrating radiations, typified by the "fallout problem" shown in figure 3 :

$\tau=\mu t=$ mean-free-path thickness of layers of material between primary source and the electron, where $\mu$ is the attenuation coefficient in reciprocal units of the linear thickness $t$,

$\sigma / 4 \pi=$ surface density of contaminant, in the case of the fallout problem, in units of photons emitted per unit time from unit surface area into unit solid angle,

$I(\tau, a, b)=I^{o}(\tau, a, b)+I^{s}(\tau a, b)$

$=$ the detector response to the unscattered and scattered components, respectively, of radiation transmitted by a slab barrier having thickness $\tau$ and a rectangular exit surface of dimensions $a, b$. 


\section{Arbitrary Detector Positions}

Although the results in this paper relate directly to the response of a detector in a corner position, the response at an arbitrary position $(\alpha, \beta)$ (shown in fig. 4 ) is of more general interest.

A compounding rule found in standard illuminating engineering texts [10] yields the detector response, $I(a, b ; \alpha, \beta)$, at such a position by combining corner-position data from four related rectangles:

$$
I(a, b ; \alpha, \beta)=I(\alpha, \beta)+I(a-\alpha, b-\beta)+I(\alpha, b-\beta)+I(a-\alpha, \beta),
$$

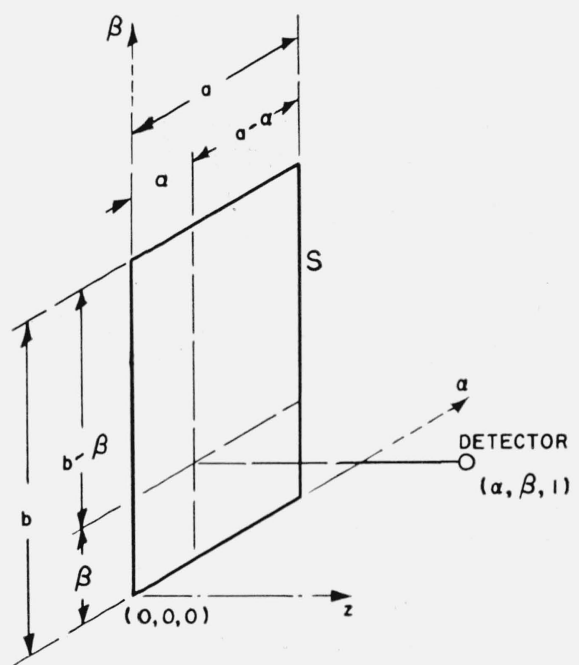

a

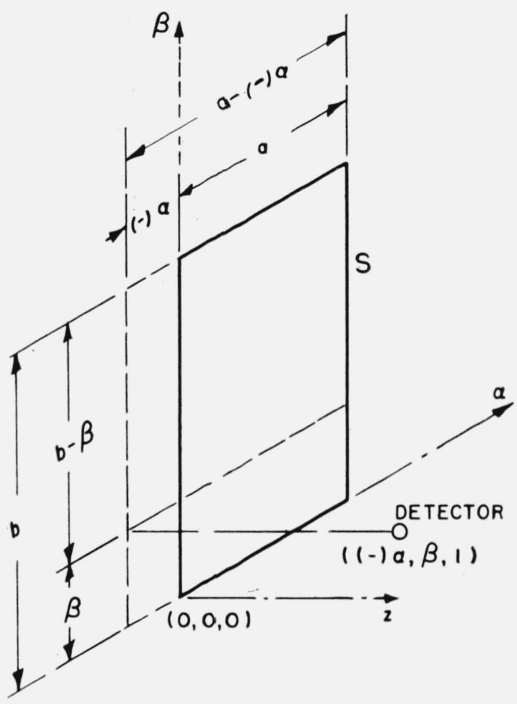

b

FIGURE 4. Examples using the scaled coordinates of figure 3, of noncorner positions (a) within the rectangular area directly under the source and (b) outside this area.

in which the terms on the right are obtained by replacing the parameters $(a, b)$ of $(6)$ or (10) by the quantities indicated. Since both (6) and (10) are odd functions in $a b$, i.e.,

$$
I( \pm a, \mp b)=-I(a, b),
$$

the rule in (11) applies not only to detector positions $0 \leq \alpha \leq a, 0 \leq \beta \leq b$ as in figure $4 \mathrm{a}$, but to any position $-\infty<\alpha<+\infty,-\infty<\beta<+\infty$ as shown in the example in figure $4 \mathrm{~b}$.

\section{Legendre Expansion of the Response Function}

\subsection{Isotropic Detector}

The primary task performed in this work is the evaluation of the Legendre expansion coefficients $p_{l}(\boldsymbol{a}, b)$ by performing the integrations indicated in (8).

Use of scaled cartesian coordinates leads to the transformations

$$
\cos \theta=h / r=1 / \sqrt{\alpha^{2}+\beta^{2}+1}
$$

and

$$
d \Omega_{S}(\theta)=d S \cdot \cos \theta / r^{2}=d \alpha d \beta \cdot\left(\alpha^{2}+\beta^{2}+1\right)^{-3 / 2}
$$

so that we may write (8) explicitly as

$$
p_{l}(a, b)=\int_{0}^{a} d \alpha \int_{0}^{b} d \beta \cdot\left(\alpha^{2}+\beta^{2}+1\right)^{-\frac{3}{2}} \cdot P_{l}\left(1 / \sqrt{\alpha^{2}+\beta^{2}+1}\right) .
$$


Evaluation of this family of double integrals is outlined in appendix A. 'The first four $p_{l}(a, b)$ 's were found to be

$$
\begin{aligned}
& p_{0}(a, b)=\tan ^{-1} \frac{a b}{\sqrt{a^{2}+b^{2}+1}}, \\
& p_{1}(a, b)=\frac{1}{2}\left\{\frac{b}{\sqrt{b^{2}+1}} \tan ^{-1} \frac{a}{\sqrt{b^{2}+1}}+\frac{a}{\sqrt{a^{2}+1}} \tan ^{-1} \frac{b}{\sqrt{a^{2}+1}}\right\} \\
& p_{2}(a, b)=\frac{1}{2} \frac{a b}{\sqrt{a^{2}+b^{2}+1}}\left\{\frac{1}{a^{2}+1}+\frac{1}{b^{2}+1}\right\},
\end{aligned}
$$

and

$$
\begin{aligned}
& p_{3}(a, b)= \frac{1}{8}\left\{-\left(\frac{b}{\sqrt{b^{2}+1}} \tan ^{-1} \frac{a}{\sqrt{b^{2}+1}}+\frac{a}{\sqrt{a^{2}+1}} \tan ^{-1} \frac{b}{\sqrt{a^{2}+1}}\right)\right. \\
&+\frac{5}{2}\left[\frac{b}{\left(b^{2}+1\right)^{\frac{3}{2}}} \tan ^{-1} \frac{a}{\sqrt{b^{2}+1}}+\frac{a}{\left(a^{2}+1\right)^{\frac{3}{2}}} \tan ^{-1} \frac{b}{\sqrt{a^{2}+1}}\right. \\
&\left.\left.+\frac{a b}{a^{2}+b^{2}+1}\left(\frac{1}{a^{2}+1}+\frac{1}{b^{2}+1}\right)\right]\right\} .
\end{aligned}
$$

The $p_{0}(a, b)$ term is the result given by Spencer [2] for a diffuse (i.e., $g(\cos \theta)=$ constant) rectangular source and isotropic detector, and $p_{1}(a, b)$ is the result given by illumination [5-8] and heat-exchange [9] engineering studies for a diffuse rectangular source and a surface-type detector parallel to the source plane.

The terms become progressively more complicated with increasing $l$, but the even and odd general terms as given by (38) and (42) in appendix A are amenable to programing for an automatic computer. Table 1 contains $p_{l}(a, b)$ 's evaluated using an IBM type 704 computer for $0 \leq l \leq 13$ over a grid of $a$ and $b$ values $0.1 \leq a \leq b \leq 20$. These may now be inserted in (6) to give the detector response $I(a, b)$ in a corner position.

These $\mathrm{p}_{l}(a, b)$ 's may be checked by reconstructing $\psi(\theta, a, b)$ according to $(7)$ and comparing with the exact results given in reference [1]. As shown in figure $5, \psi(\theta, a, b)$ is reasonably well described for the case $a=1, b=2$, neglecting terms in excess of $l=7$. If, however, higher $g_{l}$ terms are available and contain appreciable information, as for a severely peaked angular distribution, greater accuracy may be achieved by including additional terms in (6).

\subsection{Cosine Detector}

The $g_{l}$ coefficients may in principle be generated for any azimuthally independent uniform plane source by performing the integration indicated in (5) by analytic, numerical, or graphical means. Thus the Legendre expansion method might also be fruitful in similar rectangular source studies in illumination and heat-exchange engineering in which the $g(\cos \theta)$ is known. In these studies, however, the radiation field is usually described in terms of a surface-element detector ("plane detector") whose response is proportional to the cosine of the angle of incidence.

The orientation of such a plane detector may be specified by its direction $\operatorname{cosines} A, B$, and $C$ with respect to the $\alpha, \beta$, and $z$ axes. Legendre expansion coefficients $p_{l, A, B, C}(a, b)$ (analogous to the isotropic detector $p_{l}(a, b)$ 's) may each be written as the sum of three components (see ref [8], eq (17)),

$$
p_{l, A, B, C}(a, b)=A p_{l \alpha}(a, b)+B p_{l \beta}(a, b)+C p_{l z}(a, b),
$$

where $p_{l \alpha}(a, b)$ and $p_{l \beta}(a, b)$ are the Legendre coefficients for a plane detector perpendicular to $S$ and to the $\alpha$-or $\beta$-axis, respectively, and the $p_{l z}(a, b)$ 's are the coefficients for a detector parallel to $S$, i. e., perpendicular to the $z$-axis. 
TABLE 1.* Coefficients $\mathrm{p}_{l}(\mathrm{a}, \mathrm{b})$ of the Legendre expansion $(\boldsymbol{\gamma})$ of the angular response function $\psi(\theta, \mathrm{a}, \mathrm{b})$

\begin{tabular}{|c|c|c|c|c|c|c|}
\hline$l$ & $\begin{array}{l}b=0.1 \\
a=0.1\end{array}$ & $\begin{array}{l}b=0.2 \\
a=0.1\end{array}$ & $\begin{array}{l}b=0.2 \\
a=0.2\end{array}$ & $\begin{array}{l}b=0.5 \\
a=0.1\end{array}$ & $\begin{array}{l}b=0.5 \\
a=0.2\end{array}$ & $\begin{array}{l}b=0.5 \\
a=0.5\end{array}$ \\
\hline $\begin{array}{r}0 \\
1 \\
2 \\
3 \\
4 \\
5 \\
6 \\
7 \\
8 \\
9 \\
10 \\
11 \\
12 \\
13\end{array}$ & $\begin{array}{l}\text { 9. } 9012(-3) \\
9.8685 \\
9.8034 \\
9.7064 \\
9.5781 \\
9.4193 \\
9.2312 \\
9.0151 \\
8.7724 \\
8.5047 \\
\text { 8. } 2140 \\
7.9020 \\
7.5710 \\
7.2231\end{array}$ & $\begin{array}{l}1.9516(-2) \\
1.9358 \\
1.9046 \\
1.8585 \\
1.7984 \\
1.7254 \\
1.6408 \\
1.5461 \\
1.4430 \\
1.3333 \\
1.2188 \\
1.1015 \\
9.8316(-3) \\
8.6565\end{array}$ & $\begin{array}{l}3.8471(-2) \\
3.7980 \\
3.7010 \\
3.5587 \\
3.3750 \\
3.1545 \\
2.9031 \\
2.6270 \\
2.3332 \\
2.0287 \\
1.7206 \\
1.4159 \\
1.1211 \\
8.4197(-3)\end{array}$ & $\begin{array}{l}\text { 4. } 4514(-2) \\
\text { 4. } 2915 \\
3.9869 \\
3.5656 \\
3.0658 \\
2.5301 \\
2.0015 \\
1.5177 \\
1.1076 \\
7.8884(-3) \\
5.6642 \\
4.3403 \\
3.7609 \\
3.7105\end{array}$ & $\begin{array}{r}8.7819(-2) \\
8.4281 \\
7.7547 \\
6.8256 \\
5.7261 \\
4.5524 \\
3.3997 \\
2.3514 \\
1.4701 \\
7.9220(-3) \\
3.2668 \\
5.7140(-4) \\
-5.2395 \\
-4.9818\end{array}$ & $\begin{array}{r}2.0136 \quad(-1) \\
1.8807 \\
1.6330 \\
1.3035 \\
9.3443(-2) \\
5.7010 \\
2.4985 \\
2.2553(-4) \\
-1.5825(-2) \\
-2.3202 \\
-2.3214 \\
-1.8030 \\
-1.0173 \\
-2.0192(-3)\end{array}$ \\
\hline$l$ & $\begin{array}{l}b=1.0 \\
a=0.1\end{array}$ & $\begin{array}{l}b=1.0 \\
a=0.2\end{array}$ & $\begin{array}{l}b=1.0 \\
a=0.5\end{array}$ & $\begin{array}{l}b=1.0 \\
a=1.0\end{array}$ & $\begin{array}{l}b=2.0 \\
a=0.1\end{array}$ & $\begin{array}{l}b=2.0 \\
a=0.2\end{array}$ \\
\hline $\begin{array}{r}0 \\
1 \\
2 \\
3 \\
4 \\
5 \\
6 \\
7 \\
8 \\
9 \\
10 \\
11 \\
12 \\
13\end{array}$ & $\begin{array}{l}7.0418(-2) \\
6.3910 \\
5.2552 \\
3.9093 \\
2.6458 \\
1.6848 \\
1.1172 \\
8.9990(-3) \\
8.9914 \\
9.5780 \\
9.5857 \\
8.5803 \\
6.8321 \\
4.9912\end{array}$ & $\begin{array}{l}1.3912(-1) \\
1.2572 \\
1.0233 \\
7.4574(-2) \\
4.8456 \\
2.8473 \\
1.6480 \\
1.1601 \\
1.1114 \\
1.1850 \\
1.1468 \\
9.1332(-3) \\
5.4331 \\
1.6968\end{array}$ & $\begin{array}{l}3.2175(-1) \\
2.8332 \\
2.1667 \\
1.3858 \\
6.6728(-2) \\
1.4080 \\
-1.4393 \\
-2.1736 \\
-1.6157 \\
-6.8461(-3) \\
-3.5674(-4) \\
1.0787(-3) \\
-1.0131 \\
-3.4306\end{array}$ & $\begin{array}{r}5.2360(-1) \\
4.3521 \\
2.8868 \\
1.3137 \\
8.0187(-3) \\
-5.6244(-2) \\
-6.2546 \\
-3.2689 \\
4.4199(-3) \\
2.6786(-2) \\
2.7119 \\
1.1910 \\
-5.9052(-3) \\
-1.5587(-2)\end{array}$ & $\begin{array}{l}\text { 8. } 9117(-2) \\
7.4970 \\
5.3170 \\
3.2627 \\
1.9494 \\
1.4458 \\
1.3947 \\
1.3716 \\
1.1807 \\
8.9548(-3) \\
6.8459 \\
6.2105 \\
6.3528 \\
6.1304\end{array}$ & $\begin{array}{l}1.7632 \\
1.4769 \\
1.0348 \\
6.1652 \\
3.4619 \\
2.3811 \\
2.2105 \\
2.1019 \\
1.6654 \\
1.0498 \\
5.9365 \\
4.4251 \\
4.5618 \\
4.0585\end{array}$ \\
\hline$l$ & $\begin{array}{l}b=2.0 \\
a=0.5\end{array}$ & $\begin{array}{l}b=2.0 \\
a=1.0\end{array}$ & $\begin{array}{l}b=2.0 \\
a=2.0\end{array}$ & $\begin{array}{l}b=5.0 \\
a=0.1\end{array}$ & $\begin{array}{l}b=5.0 \\
a=0.2\end{array}$ & $\begin{array}{l}b=5.0 \\
a=0.5\end{array}$ \\
\hline $\begin{array}{r}0 \\
1 \\
2 \\
3 \\
4 \\
5 \\
6 \\
7 \\
8 \\
9 \\
10 \\
11 \\
12 \\
13\end{array}$ & $\begin{array}{r}4.1152(-1) \\
3.3564 \\
2.1822 \\
1.0654 \\
3.3703(-2) \\
4.3254(-3) \\
7.2294(-4) \\
1.3615(-3) \\
-3.9207 \\
-1.1888(-2) \\
-1.4791 \quad(-3) \\
-9.9201(-3) \\
-1.6638 \\
3.7038\end{array}$ & $\begin{array}{r}6.8472(-1) \\
5.2582 \\
2.8577 \\
7.0518(-2) \\
-4.0423 \\
-6.6172 \\
-2.9200 \\
8.1359(-3) \\
1.9132(-2) \\
9.8109(-3) \\
-8.7945(-4) \\
-2.6009(-3) \\
9.0735(-4) \\
1.4956(-3)\end{array}$ & $\begin{array}{r}9.2730(-1) \\
6.5269 \\
2.6667 \\
-2.6030(-2) \\
-1.2049(-1) \\
-6.0731(-2) \\
2.8998 \\
5.6423 \\
1.9243 \\
-2.5299 \\
-3.1339 \\
-3.7923(-3) \\
2.0619(-2) \\
1.7773\end{array}$ & $\begin{array}{l}\text { 9. } 7727(-2) \\
7.7896 \\
5.0420 \\
2.9189 \\
1.9463 \\
1.7051 \\
1.5548 \\
1.2639 \\
9.8612 \\
8.6130 \\
8.1909 \\
7.3200 \\
5.9839 \\
5.0339\end{array}$ & $\begin{array}{l}1.9351(-1) \\
1.5352 \\
9.7983(-2) \\
5.4792 \\
3.4566 \\
2.8992 \\
2.5295 \\
1.8859 \\
1.2767 \\
9.8248(-3) \\
8.6299 \\
6.6370 \\
3.8145 \\
1.8646\end{array}$ & $\begin{array}{r}\text { 4. } 5396(-1) \\
\text { 3. } 4997 \\
2.0456 \\
\text { 8. } 9638(-2) \\
\text { 3. } 3712 \\
\text { 1. } 7192 \\
8.4961(-3) \\
-4.1356 \\
-1.3530(-2) \\
-1.3389 \\
-8.0041(-3) \\
-4.5171 \\
-3.6896 \\
-1.7941\end{array}$ \\
\hline$l$ & $\begin{array}{l}b=5.0 \\
a=1.0\end{array}$ & $\begin{array}{l}b=5.0 \\
a=2.0\end{array}$ & $\begin{array}{l}b=5.0 \\
a=5.0\end{array}$ & $\begin{array}{l}b=10.0 \\
a=0.1\end{array}$ & $\begin{array}{l}b=10.0 \\
a=0.2\end{array}$ & $\begin{array}{l}b=10.0 \\
a=0.5\end{array}$ \\
\hline $\begin{array}{r}0 \\
1 \\
2 \\
3 \\
4 \\
5 \\
6 \\
7 \\
8 \\
9 \\
10 \\
11 \\
12 \\
13\end{array}$ & $\begin{array}{r}7.6616(-1) \\
5.5285 \\
2.5907 \\
3.8325(-2) \\
-4.7545 \\
-4.1089 \\
-1.4967 \\
-3.3765(-3) \\
6.9961(-4) \\
7.9552(-3) \\
\text { 1. } 2904(-2) \\
7.2894(-3) \\
-4.0976 \\
-9.4124\end{array}$ & $\begin{array}{r}1.0697(0) \\
6.9768(-1) \\
2.1768 \quad(-2) \\
-8.0873(-2) \\
-1.1485(-1) \\
-1.5559(-2) \\
5.0088 \\
3.2101 \\
-1.1825 \\
-2.3266 \\
-4.7751(-3) \\
9.7856 \\
6.8161 \\
-1.0631\end{array}$ & $\begin{array}{r}1.2925(0) \\
7.6053(-1) \\
1.3464 \\
-1.6607 \\
-9.3400(-2) \\
5.5039 \\
6.8498 \\
-1.4585 \\
-4.9763 \\
-4.7962(-3) \\
3.4526(-2) \\
1.4253 \\
-2.1923 \\
-1.8035\end{array}$ & $\begin{array}{l}9.9172(-2) \\
7.8117 \\
4.9749 \\
2.8872 \\
1.9878 \\
1.7418 \\
1.5308 \\
1.2260 \\
9.9606(-3) \\
8.9743 \\
8.2073 \\
7.0017 \\
5.8764 \\
5.2905\end{array}$ & $\begin{array}{l}1.9640(-1) \\
1.5396 \\
9.6643(-2) \\
5.4156 \\
3.5396 \\
2.9725 \\
2.4815 \\
1.8101 \\
1.2966 \\
1.0547 \\
8.6626(-3) \\
6.0006 \\
3.5995 \\
2.3776\end{array}$ & $\begin{array}{r}4.6117(-1) \\
3.5108 \\
2.0122 \\
8.8055(-2) \\
3.5782 \\
1.9018 \\
7.2960(-3) \\
-6.0253 \quad(-2) \\
-1.3032(-1588 \\
-1.158 \\
-7.9255(-3) \\
-6.1052 \\
-4.2231 \\
-5.1238 \quad(-4)\end{array}$ \\
\hline$l$ & $\begin{array}{l}b=10.0 \\
a=1.0\end{array}$ & $\begin{array}{l}b=10.0 \\
a=2.0\end{array}$ & $\begin{array}{l}b=10.0 \\
a=5.0\end{array}$ & $\begin{array}{l}b=10.0 \\
a=10.0\end{array}$ & $\begin{array}{l}b=20.0 \\
a=0.1\end{array}$ & $\begin{array}{l}b=20.0 \\
a=0.2\end{array}$ \\
\hline $\begin{array}{r}0 \\
1 \\
2 \\
3 \\
4 \\
5 \\
6 \\
7 \\
8 \\
9 \\
10 \\
11 \\
12 \\
13\end{array}$ & $\begin{array}{r}7.8045(-1) \\
5.5503 \\
\text { 2. } 5244 \\
\text { 3. } 5197(-2) \\
-4.3432 \\
-3.7478 \\
-1.7360 \\
-7.1162(-3) \\
1.7058 \\
\text { 1. } 1525(-2) \\
\text { 1. } 3040 \\
4.1349(-3) \\
-5.1356 \\
-6.8582\end{array}$ & $\begin{array}{r}1.0973(0) \\
7.0183(-1) \\
2.0484(-2) \\
-8.6847(-2) \\
-1.0684(-1) \\
-8.6468(-3) \\
4.5362(-2) \\
2.4916( \\
-9.7509(-3) \\
-1.6370(-2) \\
-4.6562(-3) \\
3.6459 \\
\text { 4. } 9501 \\
\text { 3. } 9658\end{array}$ & $\begin{array}{r}1.3500(0) \\
7.6864(-1) \\
1.0771 \\
-1.7179 \\
-7.6187(-2) \\
6.8721 \\
5.7789 \\
-2.9608 \\
-4.4287 \\
9.3314(-3) \\
3.3426(-2) \\
1.2963(-3) \\
-2.4409(-2) \\
-6.9441(-3)\end{array}$ & $\begin{array}{r}1.4300(0) \\
7.7903(-1) \\
6.9836(-2) \\
-1.8686(-1) \\
-5.1368(-2) \\
8.6458 \\
4.1505 \\
-4.7938 \\
-3.4798 \\
2.8212 \\
2.9638 \\
-1.6478 \\
-2.5371 \\
8.8627(-3)\end{array}$ & $\begin{array}{l}9.9544(-2) \\
7.8146 \\
4.9567 \\
2.8829 \\
2.0009 \\
1.7470 \\
1.5206 \\
1.2200 \\
1.0040 \\
9.0385(-3) \\
8.1452 \\
6.9345 \\
5.9230 \\
5.3592\end{array}$ & $\begin{array}{l}\text { 1. } 9715(-1) \\
1.5402 \quad(-2) \\
9.6278(-2) \\
5.4071 \\
3.5657 \\
2.9830 \\
2.4612 \\
1.7982 \\
1.3126 \\
1.0675 \\
8.5385(-3) \\
5.8661 \\
3.6927 \\
2.5151\end{array}$ \\
\hline
\end{tabular}

See footnote at end of table, p. 128. 
TABLE 1.* Coefficients $\mathrm{p}_{l}(\mathrm{a}, \mathrm{b})$ of the Legendre expansion $(7)$ of the angular response function $\psi(\theta, \mathrm{a}, \mathrm{b})$-Continued

\begin{tabular}{|c|c|c|c|c|c|c|}
\hline$l$ & $\begin{array}{l}b=20.0 \\
a=0.5\end{array}$ & $\begin{array}{l}b=20.0 \\
a=1.0\end{array}$ & $\begin{array}{l}b=20.0 \\
a=2.0\end{array}$ & $\begin{array}{l}b=20.0 \\
a=5.0\end{array}$ & $\begin{array}{l}b=20.0 \\
a=10.0\end{array}$ & $\begin{array}{l}b=20.0 \\
a=20.0\end{array}$ \\
\hline $\begin{array}{r}0 \\
1 \\
2 \\
3 \\
4 \\
5 \\
6 \\
7 \\
8 \\
9 \\
10 \\
11 \\
12 \\
13\end{array}$ & $\begin{array}{l}\text { 4. } 6302(-1) \\
\text { 3. } 5122 \\
2.0031 \\
8.7841(-2) \\
3.6436 \\
1.9280 \\
6.7898(-3) \\
-6.3214 \quad(-2) \\
-1.2633(-2) \\
-1.1268 \quad(-3) \\
-8.2358(-3) \\
-6.4412 \\
-3.9897 \\
-1.6924(-4)\end{array}$ & $\begin{aligned} & 7.8415(-1) \\
& 5.5532 \\
& 2.5062 \\
& 3.4772 \\
&-4.2128 \\
&-4.21) \\
&-3.6957 \\
&-1.8370 \\
&-7.7066(-3) \\
& 2.5016 \\
& 1.2164(-2) \\
& 1.2420 \\
& 3.4648(-3) \\
&-4.6694 \\
&-6.1736\end{aligned}$ & $\begin{array}{r}1.1047(0) \\
7.0240(-1) \\
2.0124(-2) \\
-8.7686(-2) \\
-1.0426(-1) \\
-7.6179(-3) \\
4.3358(-2) \\
2.3750 \\
-8.1707(-3) \\
-1.5107(-2) \\
-5.8884(-3) \\
2.3212 \\
5.8792 \\
5.3201\end{array}$ & $\begin{array}{r}1.3673(0) \\
7.6994(-1) \\
9.9215(-2) \\
-1.7372(-1) \\
-7.0077(-2) \\
7.1094 \\
5.3034 \\
-3.1702 \\
-4.0518 \\
1.2254 \\
3.0463 \\
-1.7760(-3) \\
-2.2148(-2) \\
-3.7939(-3)\end{array}$ & $\begin{aligned} & 1.4593(0) \\
& 7.8112(-1) \\
& 5.5376(-2) \\
&-1.8997(-1) \\
&-4.0924(-2) \\
& 9.0285 \\
& 3.3318 \\
&-5.2295 \\
&-2.8235 \\
& 3.2956 \\
& 2.4392 \\
&-2.1487 \\
&-2.1264 \\
& 1.4029\end{aligned}$ & $\begin{array}{r}1.5002(0) \\
7.8379(-1) \\
3.5245(-2) \\
-1.9395(-1) \\
-2.6306(-2) \\
9.5183(-2) \\
2.1754 \\
-5.7885 \\
-1.8836 \\
3.9067 \\
1.6728 \\
-2.7972 \\
-1.5087 \\
2.0756\end{array}$ \\
\hline
\end{tabular}

* Note: The figures in parenthesis in tables $1,2,3$, and 4 indicate the power of ten by which the numbers alongside and below in the same column are to be multiplied; e.g., $9.9012(-3)=0.0099012$.

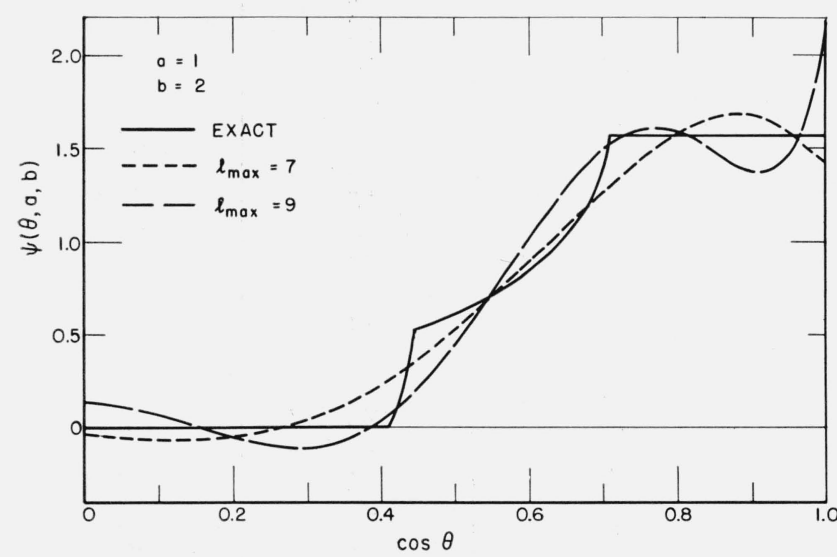

Figure 5. Reconstruction of the response function $\psi(\theta, a, b)$ according to $(10)$ for $a=b=1$ using terms $0 \leq l \leq 7$ (dotted line) and $0 \leq l \leq 9$ (dashed line). The exact function as given $\bar{b} y$ Berger and Lamkin [1] is shown as the solid line.

The dependence on the angle of incidence of radiation on a plane detector for geometries described by $p_{l \alpha}(a, b)$ and $p_{l \beta}(a, b)$ reduces to $\alpha \cos \theta$ and $\beta \cos \theta$, respectively, modifying the integrand in (15) to give

and

$$
p_{l \alpha}(a, b)=\int_{0}^{b} d \beta \int_{0}^{a} \alpha d \alpha P_{l}\left(1 / \sqrt{\left.\alpha^{2}+\beta^{2}+1\right)} /\left(\alpha^{2}+\beta^{2}+1\right)^{2}\right.
$$

$$
p_{l \beta}(a, b)=\int_{0}^{a} d \alpha \int_{0}^{b} \beta d \beta P_{l}\left(1 / \sqrt{\left.\alpha^{2}+\beta^{2}+1\right)} /\left(\alpha^{2}+\beta^{2}+1\right)^{2} .\right.
$$

Evaluation of these integrals should be similar to that for (15). In the geometry described by the $p_{l z}(a, b)$ 's, the angles of emission and incidence are identical, so that the integrand in (15) requires only an additional factor of $\cos \theta$. Thus, using the relation

$$
\cos \theta P_{l}(\cos \theta)=\left\{l P_{l-1}(\cos \theta)+(l+1) P_{l+1}(\cos \theta)\right\} /(2 l+1),
$$

the $p_{l z}(a, b)$ 's may be obtained from the $p_{l}(a, b)$ 's in table 1 as

$$
P_{l z}(a, b)=\left\{l p_{l-1}(a, b)+(l+1) p_{l+1}(a, b)\right\} /(2 l+1) .
$$

\section{Unscattered Radiation Component}

In cases involving penetrating radiations, such as gamma rays from the plane source shown in figure 3 , it is usually desirable to make a separate calculation of the unscattered radiation component, $I^{o}(\tau, a, b)$. Two reasons for this separation of components are: 
(a) In contrast to the scattered radiation component, $I^{s}(\tau, a, b)$, the unscattered component, $I^{\circ}(\tau, a, b)$, is analytic in nature and independent of the barrier material, so that it may be calculated once and for all for the relevant parameters $\tau, a$, and $b$.

(b) Since the angular distribution, $g^{\circ}(\tau, \cos \theta)$, of unscattered radiation from a plane isotropic source as expressed in (9) tends toward $\frac{\sigma}{4 \pi}(1 / \cos \theta)$ as $\tau$ becomes small, representation as a Legendre expansion in positive powers of $\cos \theta$ becomes impractical. This difficulty usually does not arise for the scattered component.

\section{1. $\tau \gtrsim 1:$ Legendre Expansion}

In order to examine the behavior discussed in (b), above, $g_{l}{ }^{\circ}(\tau)$ 's were evaluated by substituting (9) into (5). The result

$$
g_{l}{ }^{o}(\tau)=\frac{\sigma}{4 \pi}\left\{P_{l} E_{j+1}(\tau)\right\}
$$

where $E_{j}(\tau)$ is the exponential integral [11]

$$
E_{j}(\tau)=\int_{1}^{\infty} e^{-\tau \mu} u^{-j} d u
$$

and $P_{l}\left\{E_{j+1}(\tau)\right\}$ is obtained by replacing $(\cos \theta)^{j}$ in $P_{l}(\cos \theta)$ by $E_{j+1}(\tau)$. Since these $g_{l}{ }^{o}(\tau)$, although of fairly general interest, do not seem to appear explicitly in the literature, a tabulation of the quantity $\frac{4 \pi}{\sigma}\left(l+\frac{1}{2}\right) g_{l}{ }^{o}(\tau)$, (i.e., $\left.\left(l+\frac{1}{2}\right) P_{l}\left\{E_{j+1}(\tau)\right\}\right)$, for $0 \leq l \leq 13$ and $0.001 \leq \tau \leq 100$ is presented in table 2 .

A check on the values in table 2 is shown in figure 6 , in which $(4 \pi / \sigma) e^{\tau} g^{o}(\tau, \cos \theta)$, reconstructed according to (4) for $0 \leq l \leq 7$ and $0.2 \leq \tau \leq 10$, are indicated by the dotted curves. Comparison may now be made with similar results from the exact expression (9), shown by the

TABLe 2. Coefficients $(4 \pi / \sigma)\left(l+\frac{1}{2}\right) \mathrm{g}_{l}^{o}(\tau)$ of a Legendre expansion (4) of the angular distribution $(4 \pi / \sigma) \mathrm{g}^{\circ}(\tau, \cos \theta)$ of unscattered radiation from a plane isotropic source

The factor $4 \pi / \sigma$ implies unit source strength per steradian per unit of source area, and $(l+1 / 2)$ is included to allow cumulative multiplication

\begin{tabular}{|c|c|c|c|c|c|c|}
\hline & 0.001 & 0.01 & 0.1 & 0.2 & 0.5 & 1.0 \\
\hline $\begin{array}{r}0 \\
1 \\
2 \\
3 \\
4 \\
5 \\
6 \\
7 \\
8 \\
9 \\
10 \\
11 \\
12 \\
13\end{array}$ & $\begin{array}{r}3.1943(0) \\
1.4889 \\
-6.1143 \\
-2.2989 \\
7.2752 \\
2.8703 \\
-8.0184 \\
-3.3335 \\
8.5595 \\
3.7303 \\
-8.9790 \\
-4.0810 \\
9.3161 \\
4.3971\end{array}$ & $\begin{array}{r}2.0190(0) \\
1.4245 \\
-3.2090 \\
-2.1124 \\
3.3973 \\
2.5450 \\
-3.4034 \\
-2.8664 \\
3.3377 \\
3.1040 \\
-3.2372 \\
-3.2983 \\
3.1186 \\
3.4559\end{array}$ & $\begin{array}{r}9.1145(-1) \\
1.0838(0) \\
-7.1755(-1) \\
-1.2757(0) \\
3.6314(-1) \\
1.2524(0) \\
-9.6551(-2) \\
-1.1624(0) \\
-9.7963(-2) \\
1.0492(0) \\
2.3859(-1) \\
-9.3042 \\
-3.3868 \\
8.1428\end{array}$ & $\begin{array}{r}6.1135(-1) \\
8.6130 \\
-2.0852 \\
-8.3188 \\
-9.1715(-2) \\
6.7606(-1) \\
2.4954 \\
-5.1631 \\
-3.2542 \\
3.7667 \\
3.5348 \\
-2.6113 \\
-3.5306 \\
3.3172\end{array}$ & $\begin{array}{r}2.7989(-1) \\
4.8996 \\
1.3130 \\
-2.6901 \\
-2.1534 \\
1.0298 \\
1.9507 \\
-8.9640(-3) \\
-1.4873(-1) \\
-3.8228(-2) \\
1.0293(-1) \\
5.7819(-2) \\
-6.4944 \\
-6.1991\end{array}$ & $\begin{array}{r}\text { 1. } 0970(-1) \\
2.2275 \\
\text { 1. } 3712 \\
-2.6555(-2) \\
-9.3776 \\
-3.3952 \\
\text { 4. } 0965 \\
\text { 3. } 9813 \\
-9.6373(-3) \\
-3.0111(-2) \\
-4.9996(-3) \\
1.8731(-2) \\
1.0139(-3) \\
-9.7479(-3)\end{array}$ \\
\hline & 2.0 & 5.0 & 10.0 & 15.0 & 20.0 & 100.0 \\
\hline $\begin{array}{r}0 \\
1 \\
2 \\
3 \\
4 \\
5 \\
6 \\
7 \\
8 \\
9 \\
10 \\
11 \\
12 \\
13\end{array}$ & $\begin{array}{r}2.4451 \quad(-2) \\
5.6301 \\
5.1875 \\
2.1895 \\
-6.1965(-3) \\
-1.4221(-2) \\
-5.7195(-3) \\
3.9476 \\
5.1606 \\
5.1102(-4) \\
-2.6394 \\
-1.5451 \\
8.7741 \quad \\
1.2960(-3)\end{array}$ & $\begin{array}{r}5.7415(-4) \\
1.4947(-3) \\
1.8564 \\
1.6196 \\
1.0195 \\
3.9434(-4) \\
-1.1402(-5) \\
-1.4339(-4) \\
-9.8609(-5) \\
-1.3120 \\
3.0530 \\
2.4977 \\
3.8280(-6) \\
-9.5729(-6)\end{array}$ & $\begin{array}{r}2.0785(-6) \\
5.7483 \\
\text { 8. } 1118 \\
8.8022 \\
7.9587 \\
6.1188 \\
3.9668 \\
2.0817 \\
9.1367(-7) \\
8.0998(-8) \\
-1.5133(-7) \\
-1.4134 \quad \\
-5.0961(-8) \\
4.5106(-9)\end{array}$ & $\begin{array}{r}9.5930(-9) \\
2.7162(-8) \\
4.0293 \\
4.7268 \\
4.7804 \\
4.2970 \\
3.4702 \\
2.5187 \\
1.6300 \\
9.1882(-9) \\
4.3555 \\
1.4721 \\
1.6851(-10) \\
-2.5223(-11)\end{array}$ & $\begin{array}{l}4.9178(-11) \\
1.4107 \quad(-10) \\
2.1490 \\
2.6263 \\
2.8115 \\
2.7243 \\
2.4242 \\
1.9934 \\
1.5167 \\
1.0653 \\
6.8479(-11) \\
4.0174 \\
2.0205 \\
9.8335(-12)\end{array}$ & $\begin{array}{l}\text { 1. } 8418 \quad(-46) \\
\text { 5. } 4717 \\
\text { 8. } 9433 \\
\text { 1. } 2159 \quad(-45) \\
\text { 1. } 5034 \\
\text { 1. } 7498 \\
\text { 1. } 9500 \\
2.1009 \\
2.2013 \\
\text { 2. } 2522 \\
2.2561 \\
2.2172 \\
2.1418 \\
2.0335\end{array}$ \\
\hline
\end{tabular}
directly with the $p_{l}(a, b)$ 's (see table 1 ) according to $(6)$. 


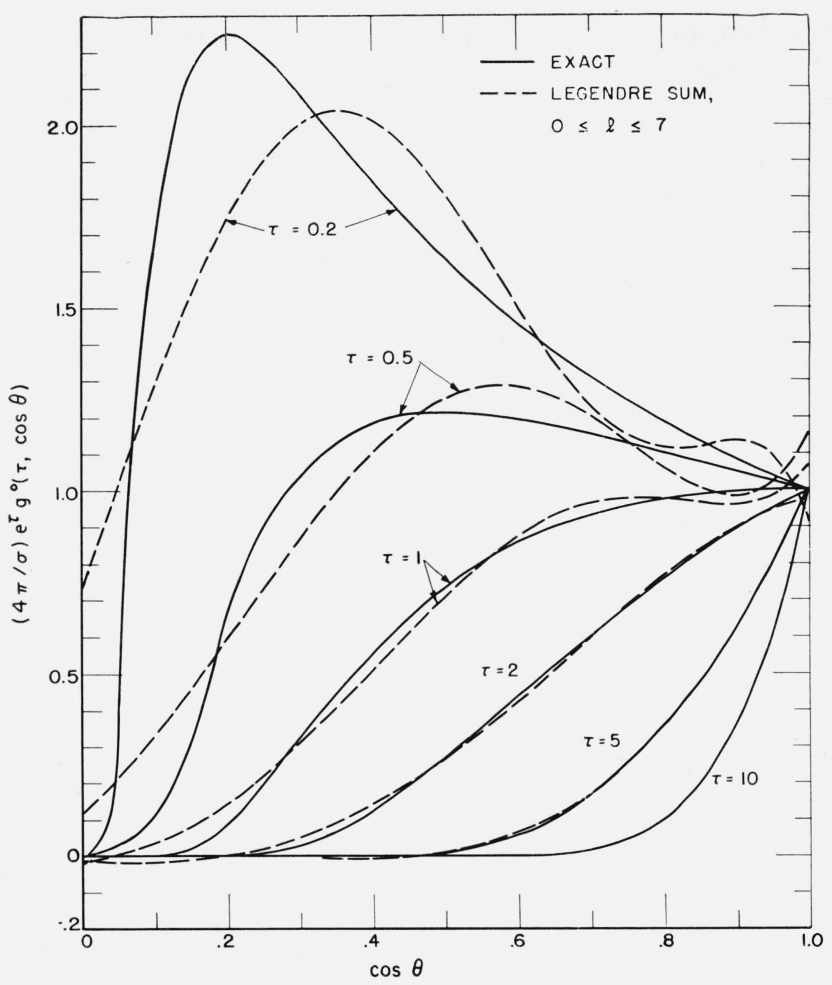

FIGURE 6. The angular distribution of unscattered radiation from a plane isotropic source reconstructed according to (4) and (25) (dotted lines) as compared with the exact expression (9) (solid lines) for $0.2 \leq \tau \leq 10$.

solid curves. From both table 2 and figure 6 it can be seen that the Legendre expansion approach, using tables 1 and 2, should give reliable results for unscattered as well as scattered radiation in the region $1 \lesssim \tau \lesssim 15$.

\section{2. $\tau \lesssim 1:$ Power Series Expansion}

The parameter $\tau$ may in some cases be very small, and for this reason it seemed desirable to find a method for evaluating $I^{o}(\tau, a, b)$, i.e.,

$$
I^{o}(\tau, a, b)=\frac{\sigma}{4 \pi} \int_{S}\left(d S / r^{2}\right) \exp (-\tau / \cos \theta),
$$

for small $\tau$. No success was had in obtaining a closed-form result, but $q_{n}(a, b)$ coefficients of the $\tau^{n}$ power series in (10) were evaluated (see appendix B) which provide adequate convergence of (10) from $\tau=0$ up to values of $\tau$ for which the $g_{l}^{o}(a, b)$ 's converge rapidly.

The first five $q_{n}(a, b)$ terms are

$$
q_{0}(a, b)=\frac{\pi}{2} \sinh ^{-1} a-\frac{b}{a} \sum_{i=0}^{\infty} \frac{1}{2 i+1}\left(\frac{a^{2}}{a^{2}+1}\right)^{i+1}\left\{\frac{\sqrt{a^{2}+1}}{b} \tan ^{-1} \frac{\sqrt{a^{2}+1}}{b}-\sum_{j=0}^{i-1} \frac{2^{2 j}(j !)^{2}}{(2 j+1) !}\left(\frac{a^{2}+1}{a^{2}+b^{2}+1}\right)^{j+1}\right\},
$$

in which the inner sum over $j$ vanishes for $i=0$,

$$
\begin{gathered}
q_{1}(a, b)=-\left\{b \sinh ^{-1} \frac{a}{\sqrt{b^{2}+1}}+a \sinh ^{-1} \frac{b}{\sqrt{a^{2}+1}}-\tan ^{-1} \frac{a b}{\sqrt{a^{2}+b^{2}+1}}\right\} \\
q_{2}(a, b)=\frac{a b}{2 !} \\
q_{3}(a, b)=-\frac{1}{3 !}\left\{-\frac{q_{1}(a, b)}{3}+\frac{1}{2 \cdot 3}\left[b\left(b^{2}+1\right) \sinh ^{-1} \frac{a}{\sqrt{b^{2}+1}}+a\left(a^{2}+1\right) \sinh ^{-1} \frac{b}{\sqrt{a^{2}+1}}\right]+\frac{a b \sqrt{a^{2}+b^{2}+1}}{3}\right\},
\end{gathered}
$$


and

$$
q_{4}(a, b)=\frac{1}{4 !}\left\{(2 !) q_{2}(a, b)+\frac{a^{3} b}{3 \cdot 1}+\frac{a b^{3}}{1 \cdot 3}\right\}
$$

For large $a$ and $b,(a \leq b)$, the expression within the braces in (28) becomes small very quickly with increasing $i$. Indeed, over the entire range of $a$ and $b$, i.e., $0 \leq a \leq b \leq \infty$, summation over $i$ from $i=0$ to $i=6$ gives values for $q_{0}(a, b)$ departing less than 0.01 percent from their respective values when the summation is carried to $i>20$. A partial check of (28) is obtained by noting that the series part of the expression vanishes for large $b$, that is,

$$
\lim _{b \rightarrow \infty} q_{0}(a, b)=\frac{\pi}{2} \sinh ^{-1} a,
$$

in agreement with the result of Andrews [12] for the detector response to an infinite strip source in a nonattenuating medium.

The remaining $q_{n}(a, b)$ terms form two distinct sets, as did the $p_{l}(a, b)$ 's, depending on whether $n$ is even or odd. The general terms for these sets are given in (57) and (58) of appendix B.

Values of $I^{o}(\tau, a, b) 4 \pi / \sigma$ for $0.01 \leq \tau \leq 10$ and $0.1 \leq a \leq b \leq 20$, as evaluated with the help of an IBM 704 computer, are presented in table 4. Numbers preceded by asterisks were evaluated using the Legendre expansion approach (15) and (25) for $0 \leq l \leq 13$, and the others using the $\tau^{n}$ series (10) for $0 \leq n \leq 20$.

Since evaluation of (10) for small specific values of $\tau$ may be faster than interpolation from table $4, q_{n}(a, b)$ is given in table 3 for $0 \leq n \leq 9$ and $0.1<a<b<20$.

TABLE 3. Coefficients $\mathrm{q}_{\mathrm{n}}(\mathrm{a}, \mathrm{b})$ of the $\tau^{\mathrm{n}}$ power series expansion $(10)$ of the corner position flux $\mathrm{I}^{\circ}$ ( $\left.\tau, \mathrm{a}, \mathrm{b}\right)$ of un-

\begin{tabular}{|c|c|c|c|c|c|c|}
\hline$n$ & $\begin{array}{l}b=0.1 \\
a=0.1\end{array}$ & $\begin{array}{l}b=0.2 \\
a=0.1\end{array}$ & $\begin{array}{l}b=0.2 \\
a=0.2\end{array}$ & $\begin{array}{l}b=0.5 \\
a=0.1\end{array}$ & $\begin{array}{l}b=0.5 \\
a=0.2\end{array}$ & $\begin{array}{l}b=0.5 \\
a=0.5\end{array}$ \\
\hline $\begin{array}{l}0 \\
1 \\
2 \\
3 \\
4 \\
5 \\
6 \\
7 \\
8 \\
9\end{array}$ & $\begin{array}{r}9.9340(-3) \\
-9.9669 \\
5.0000 \\
-1.6722 \\
4.1944(-4) \\
-8.4169(-5) \\
1.4075(-6) \\
-2.0174(-6) \\
2.5302(-7) \\
-2.8208(-8)\end{array}$ & $\begin{array}{r}1.9675(-2) \\
-1.9836 \\
10.0000(-3) \\
-3.3609 \\
8.4722(-4) \\
-1.7086 \\
2.8716(-5) \\
-4.1368(-6) \\
5.2148(-7) \\
-5.8435(-8)\end{array}$ & $\begin{array}{r}\text { 3. } 8971(-2) \\
-3.9481 \\
2.0000 \\
-6.7547(-3) \\
1.7111 \\
-3.4679(-4) \\
5.8574(-5) \\
-8.4805(-6) \\
1.0744 \quad \\
-1.2101(-7)\end{array}$ & $\begin{array}{r}4.6222(-2) \\
-4.8047 \\
2.5000 \\
-8.6819(-3) \\
2.2639 \\
-4.7282(-4) \\
8.2390(-5) \\
-1.2320 \\
1.6141(-6) \\
-1.8819(-7)\end{array}$ & $\begin{array}{r}9.1603(-2) \\
-9.5656 \\
5.0000 \\
-1.7443 \\
4.5694(-3) \\
-9.5873(-4) \\
1.6783(-5) \\
-2.5213(-5) \\
3.3183(-6) \\
-3.8869(-7)\end{array}$ & $\begin{array}{r}2.1600(-1) \\
-2.3215 \\
1.2500 \\
-4.4960(-2) \\
1.2153 \\
-2.6333(-3) \\
4.7647(-4) \\
-7.4048(-5) \\
1.0090 \\
-1.2248(-6)\end{array}$ \\
\hline$n$ & $\begin{array}{l}b=1.0 \\
a=0.1\end{array}$ & $\begin{array}{l}b=1.0 \\
a=0.2\end{array}$ & $\begin{array}{l}b=1.0 \\
a=0.5\end{array}$ & $\begin{array}{l}b=1.0 \\
a=1.0\end{array}$ & $\begin{array}{l}b=2.0 \\
a=0.1\end{array}$ & $\begin{array}{l}b=2.0 \\
a=0.2\end{array}$ \\
\hline $\begin{array}{l}0 \\
1 \\
2 \\
3 \\
4 \\
5 \\
6 \\
7 \\
8 \\
9\end{array}$ & $\begin{array}{r}7.8327(-2) \\
-8.8020 \\
5.0000 \\
-1.9154 \\
5.5694(-3) \\
-1.3114(-4) \\
2.6050(-4) \\
-4.4892(-5) \\
6.8492(-6) \\
-9.3939(-7)\end{array}$ & $\begin{array}{r}1.5540(-1) \\
-1.7535 \\
10.0000(-2) \\
-3.8455 \\
1.1222 \\
-2.6517(-3) \\
5.2848(-4) \\
-9.1365(-5) \\
1.3982 \\
-1.9233(-6)\end{array}$ & $\begin{array}{r}3.6887(-1) \\
-4.2718 \\
2.5000 \\
-9.8624(-2) \\
2.9514 \quad \\
-7.1478(-3) \\
1.4593 \\
-2.5829(-4) \\
4.0448(-5) \\
-5.6906(-6)\end{array}$ & $\begin{array}{r}6.3951(-1) \\
-7.9336 \\
5.0000 \\
-2.1346 \\
6.9444(-2) \\
-1.8357 \\
4.1049(-3) \\
-7.9826(-4) \\
1.3771 \\
-2.1392(-5)\end{array}$ & $\begin{array}{r}1.1046(-1) \\
-1.4421 \\
10.0000(-2) \\
-4.9338 \\
1.9472 \\
-6.5195(-3) \\
1.9117 \\
-5.0012(-4) \\
1.1811 \\
-2.5399(-5)\end{array}$ & $\begin{array}{r}2.1946(-1) \\
-2.8755 \\
2.0000 \\
-9.8916(-2) \\
3.9111 \\
-1.3113 \\
3.8496(-3) \\
-1.0081 \\
2.3829(-4) \\
-5.1290(-5)\end{array}$ \\
\hline$n$ & $\begin{array}{l}b=2.0 \\
a=0.5\end{array}$ & $\begin{array}{l}b=2.0 \\
a=1.0\end{array}$ & $\begin{array}{l}b=2.0 \\
a=2.0\end{array}$ & $\begin{array}{l}b=5.0 \\
a=0.1\end{array}$ & $\begin{array}{l}b=5.0 \\
a=0.2\end{array}$ & $\begin{array}{l}b=5.0 \\
a=0.5\end{array}$ \\
\hline $\begin{array}{l}0 \\
1 \\
2 \\
3 \\
4 \\
5 \\
6 \\
7 \\
8 \\
9\end{array}$ & $\begin{array}{r}5.2536(-1) \\
-7.0456 \\
5.0000 \\
-2.5139 \\
1.0069 \\
-3.4104(-2) \\
1.0095 \\
-2.6625(-3) \\
6.3366(-4) \\
-1.3730\end{array}$ & $\begin{array}{r}9.3072(-1) \\
-1.3285(0) \\
1.0000 \\
-5.3007(-1) \\
2.2222 \\
-7.8243(-2) \\
2.3951 \\
-6.5116(-3) \\
1.5949 \\
-3.5550(-4)\end{array}$ & $\begin{array}{r}1.4074(0) \\
-2.2916 \\
2.0000 \\
-1.2410 \\
6.1111(-1) \\
-2.5253 \\
9.0494(-2) \\
-2.8734 \\
8.2096(-3) \\
-2.1351\end{array}$ & $\begin{array}{r}1.3708(-1) \\
-2.3108 \\
2.5000 \\
-2.3179 \\
1.9451 \\
-1.4685 \\
9.9117(-2) \\
-5.9935 \\
3.2671 \\
-1.6173\end{array}$ & $\begin{array}{r}2.7263(-1) \\
-4.6120 \\
5.0000 \\
-4.6397 \\
3.8944 \\
-2.9404 \\
1.9849 \\
-1.2004 \\
6.5448(-2) \\
-3.2405\end{array}$ & $\begin{array}{r}6.5729(-1) \\
-1.1372(0) \\
1.2500 \\
-1.1666 \\
9.8090 \\
-7.4123 \\
5.0081 \\
-3.0320 \\
1.6550 \\
-8.2045(-2)\end{array}$ \\
\hline
\end{tabular}
scattered radiation from a rectangular plane isotropic source 
TABLE 3. Coefficients $\mathrm{q}_{\mathrm{n}}(\mathrm{a}, \mathrm{b})$ of the $\tau^{\mathrm{n}}$ power series expansion (10) of the corner position flux $\mathrm{I}^{\circ}(\tau, \mathrm{a}, \mathrm{b})$ of unscattered radiation from a rectangular plane isotropic source-Continued

\begin{tabular}{|c|c|c|c|c|c|c|}
\hline$n$ & $\begin{array}{l}b=5.0 \\
a=1.0\end{array}$ & $\begin{array}{l}b=5.0 \\
a=2.0\end{array}$ & $\begin{array}{l}b=5.0 \\
a=5.0\end{array}$ & $\begin{array}{l}b=10.0 \\
a=0.1\end{array}$ & $\begin{array}{l}b=10.0 \\
a=0.2\end{array}$ & $\begin{array}{l}b=10.0 \\
a=0.5\end{array}$ \\
\hline $\begin{array}{l}0 \\
1 \\
2 \\
3 \\
4 \\
5 \\
6 \\
7 \\
8 \\
9 \\
\end{array}$ & $\begin{array}{r}1.1879(0) \\
-2.1837 \\
2.5000 \\
-2.3782 \\
2.0139 \\
-1.5272 \\
1.0353 \\
-6.2923(-1) \\
3.4493 \\
-1.7178 \\
\end{array}$ & $\begin{array}{r}1.8793(0) \\
-3.9333 \\
5.0000 \\
-5.0728 \\
4.4444 \\
-3.4397 \\
2.3716 \\
-1.4665 \\
8.1909(-1) \\
-4.1614 \\
\end{array}$ & $\begin{array}{r}2.7262(0) \\
-7.3832 \\
1.2500(1) \\
-1.6595 \\
1.8403 \\
-1.7554 \\
1.4695 \\
-1.0971 \\
7.3983(0) \\
-4.5541 \\
\end{array}$ & $\begin{array}{r}1.4685(-1) \\
-2.9966 \\
5.0000 \\
-8.6256 \\
1.4307 \quad(0) \\
-2.1472 \\
2.8721 \\
-3.4332 \\
3.6949 \\
-3.6090 \\
\end{array}$ & $\begin{array}{r}2.9217(-1) \\
-5.9833 \\
10.0000 \\
-1.7256(0) \\
2.8622 \\
-4.2957 \\
5.7461 \\
-6.8689 \\
7.3929 \\
-7.2214 \\
\end{array}$ & $\begin{array}{r}7.0607(-1) \\
-1.4798(0) \\
2.5000 \\
-4.3227 \\
7.1701 \\
-1.0762(1) \\
1.4399 \\
-1.7217 \\
1.8536 \\
-1.8112 \\
\end{array}$ \\
\hline$n$ & $\begin{array}{l}b=10.0 \\
a=1.0\end{array}$ & $\begin{array}{l}b=10.0 \\
a=2.0\end{array}$ & $\begin{array}{l}b=10.0 \\
a=5.0\end{array}$ & $\begin{array}{l}b=10.0 \\
a=10.0\end{array}$ & $\begin{array}{l}b=20.0 \\
a=0.1\end{array}$ & $\begin{array}{l}b=20.0 \\
a=0.2\end{array}$ \\
\hline $\begin{array}{l}0 \\
1 \\
2 \\
3 \\
4 \\
5 \\
6 \\
7 \\
8 \\
9 \\
\end{array}$ & $\begin{array}{r}1.2849(0) \\
-2.8671 \\
5.0000 \\
-8.7046 \\
1.4444(1) \\
-2.1687 \\
2.9038 \\
-3.4758 \\
3.7462 \\
-3.6647 \\
\end{array}$ & $\begin{array}{r}2.0692(0) \\
-5.2864 \\
1.0000(1) \\
-1.7838 \\
2.9722 \\
-4.4719 \\
6.0067 \\
-7.2190 \\
7.8159 \\
-7.6824 \\
\end{array}$ & $\begin{array}{r}3.1467(0) \\
-1.0571(1) \\
2.5000 \\
-5.0356 \\
8.8889 \\
-1.3854(2) \\
1.9201 \\
-2.3848 \\
2.6756 \\
-2.7323 \\
\end{array}$ & $\begin{array}{r}3.7961 \quad(0) \\
-1.6127(1) \\
5.0000 \\
-1.2892(2) \\
2.8194 \\
-5.3227 \\
8.8285 \\
-1.3066(3) \\
1.7475 \\
-2.1341 \\
\end{array}$ & $\begin{array}{r}1.5182(-1) \\
-3.6878 \\
10.0000 \\
-3.3683(0) \\
1.1195(1) \\
-3.3586 \\
8.9635 \\
-2.1364(2) \\
4.5831 \\
-8.9221 \\
\end{array}$ & $\begin{array}{r}3.0211(-1) \\
-7.3658 \\
2.0000(0) \\
-6.7373 \\
2.2391(1) \\
-6.7176 \\
1.7928(2) \\
-4.2732 \\
9.1672 \\
-1.7846(3) \\
\end{array}$ \\
\hline$n$ & $\begin{array}{l}b=20.0 \\
a=0.5\end{array}$ & $\begin{array}{l}b=20.0 \\
a=1.0\end{array}$ & $\begin{array}{l}b=20.0 \\
a=2.0\end{array}$ & $\begin{array}{l}b=20.0 \\
a=5.0\end{array}$ & $\begin{array}{l}b=20.0 \\
a=10.0\end{array}$ & $\begin{array}{l}b=20.0 \\
a=20.0\end{array}$ \\
\hline $\begin{array}{l}0 \\
1 \\
2 \\
3 \\
4 \\
5 \\
6 \\
7 \\
8 \\
9\end{array}$ & $\begin{array}{r}7.3091(-1) \\
-1.8253(0) \\
5.0000 \\
-1.6854(1) \\
5.6007 \\
-1.6803(2) \\
4.4847 \\
-1.0690(3) \\
2.2935 \\
-4.4652 \\
\end{array}$ & $\begin{array}{r}1.3345(0) \\
-3.5577 \\
1.0000(1) \\
-3.3781 \\
1.1222(2) \\
-3.3669 \\
8.9882 \\
-2.1430 \\
4.5990 \\
-8.9564\end{array}$ & $\begin{array}{r}2.1679(0) \\
-6.6641 \\
2.0000(1) \\
-6.8107 \\
2.2611(2) \\
-6.7850 \\
1.8127(3) \\
-4.3264 \\
9.2953 \\
-1.8123(4)\end{array}$ & $\begin{array}{r}3.3843(0) \\
-1.3955(1) \\
5.0000 \\
-1.7800(2) \\
5.9444 \\
-1.7901(3) \\
4.8099 \\
-1.1564(4) \\
2.5046 \\
-4.9253\end{array}$ & $\begin{array}{r}4.2228(0) \\
-2.2546(1) \\
1.0000(2) \\
-3.9741 \\
1.3972(3) \\
-4.3433 \\
1.2006(4) \\
-2.9741 \\
6.6542 \\
-1.3550(5)\end{array}$ & $\begin{array}{r}4.8801(0) \\
-3.3719(1) \\
2.0000(2) \\
-1.0231(3) \\
4.4611 \\
-1.6801(4) \\
5.5605 \\
-1.6422(5) \\
4.3835 \\
-1.0685(6)\end{array}$ \\
\hline
\end{tabular}

TABLE 4. Unscattered flux $(4 \pi / \sigma) \mathrm{I}^{\circ}(\tau, a, b)$ of radiation, at a corner position, from a rectangular plane isotropic source calculated using tables 1 and 2 (numbers preceded by asterisks) and using table 3 (no asterisks)

\begin{tabular}{|c|c|c|c|c|c|c|}
\hline$\tau$ & $\begin{array}{l}b=0.1 \\
a=0.1\end{array}$ & $\begin{array}{l}b=0.2 \\
a=0.1\end{array}$ & $\begin{array}{l}b=0.2 \\
a=0.2\end{array}$ & $\begin{array}{l}b=0.5 \\
a=0.1\end{array}$ & $\begin{array}{l}b=0.5 \\
a=0.2\end{array}$ & $\begin{array}{l}b=0.5 \\
a=0.5\end{array}$ \\
\hline $\begin{array}{l}0 \\
0.01 \\
0.02 \\
0.05 \\
0.1\end{array}$ & $\begin{array}{l}9.93(-3) \\
9.83 \\
9.74 \\
9.44 \\
8.99\end{array}$ & $\begin{array}{l}1.97(-2) \\
1.95 \\
1.93 \\
1.87 \\
1.78\end{array}$ & $\begin{array}{l}3.90(-2) \\
3.86 \\
3.82 \\
3.70 \\
3.52\end{array}$ & $\begin{array}{l}\text { 4. } 62(-2) \\
4.57 \\
4.53 \\
4.39 \\
4.17\end{array}$ & $\begin{array}{l}9.16(-2) \\
9.07 \\
8.97 \\
8.69 \\
8.25\end{array}$ & $\begin{array}{l}2.16(-1) \\
2.14 \\
2.11 \\
2.05 \\
1.94\end{array}$ \\
\hline 0.2 & $\begin{array}{r}8.13 \\
* 8.48\end{array}$ & $\begin{array}{r}1.61 \\
* 1.65\end{array}$ & $\begin{array}{r}3.18 \\
* 3.21\end{array}$ & $\begin{array}{r}3.75 \\
* 3.78\end{array}$ & $\begin{array}{r}7.43 \\
* 7.44\end{array}$ & $\begin{array}{r}1.74 \\
*_{1} .75\end{array}$ \\
\hline 0.5 & $\begin{array}{r}6.02 \\
* 5.63\end{array}$ & $\begin{array}{r}1.19 \\
* 1.15\end{array}$ & $\begin{array}{r}2.35 \\
{ }^{*} 2.31\end{array}$ & $\begin{array}{r}2.75 \\
*_{2} .72\end{array}$ & $\begin{array}{r}5.44 \\
* 5.44\end{array}$ & $\begin{array}{r}\text { 1. } 26 \\
* 1.26\end{array}$ \\
\hline 1. 0 & $\begin{array}{r}3.64 \\
* 3.66\end{array}$ & $\begin{array}{r}7.18(-3) \\
* 7.02\end{array}$ & $\begin{array}{l}1.42(-2) \\
* 1.42\end{array}$ & $\begin{array}{r}1.64 \\
*_{1} .64\end{array}$ & $\begin{array}{r}\text { 3. } 23 \\
* 3.23\end{array}$ & $\begin{array}{r}7.38 \\
* 7.38\end{array}$ \\
\hline 2. 0 & $\begin{array}{l}1.34 \\
* 1.37\end{array}$ & $\begin{array}{l}2.62 \\
* 2.67\end{array}$ & $\begin{array}{r}5.14(-3) \\
* 5.20\end{array}$ & $\begin{array}{r}5.79 \\
* 5.82\end{array}$ & $\begin{array}{l}1.14 \\
{ }^{*} 1.14\end{array}$ & $\begin{array}{r}2.53 \\
* 2.54\end{array}$ \\
\hline 5.0 & $\begin{array}{r}6.60 \\
* 6.59\end{array}$ & $\begin{array}{l}\text { 1. } 28 \\
\text { *1. } 27\end{array}$ & ${ }^{2.47} 1.94(-4)$ & $\begin{array}{r}2.60 \\
*_{2} .59\end{array}(-4)$ & $\begin{array}{l}5.04(-4) \\
* 5.02\end{array}$ & $\begin{array}{l}1.04 \\
* 1.03\end{array}$ \\
\hline 10.0 & *4. $37(-7)$ & *8. $26(-7)$ & ${ }^{*} 1.54(-6)$ & $* 1.50(-6)$ & $* 2.83(-6)$ & $* 5.15(-6)$ \\
\hline$\tau$ & $\begin{array}{l}b=1.0 \\
a=0.1\end{array}$ & $\begin{array}{l}b=1.0 \\
a=0.2\end{array}$ & $\begin{array}{l}b=1.0 \\
a=0.5\end{array}$ & $\begin{array}{l}b=1.0 \\
a=1.0\end{array}$ & $\begin{array}{l}b=2.0 \\
a=0.1\end{array}$ & $\begin{array}{l}b=2.0 \\
a=0.2\end{array}$ \\
\hline $\begin{array}{l}0 \\
0.01 \\
0.02 \\
0.05 \\
0.1\end{array}$ & $\begin{array}{l}7.83(-2) \\
7.75 \\
7.66 \\
7.40 \\
7.00\end{array}$ & $\begin{array}{l}\text { 1. } 55(-1) \\
\text { 1. } 54 \\
\text { 1. } 52 \\
\text { 1. } 47 \\
\text { 1. } 39\end{array}$ & $\begin{array}{l}\text { 3. } 69(-1) \\
3.65 \\
3.60 \\
3.48 \\
\text { 3. } 29\end{array}$ & $\begin{array}{l}6.40(-1) \\
6.32 \\
6.24 \\
6.01 \\
5.65\end{array}$ & $\begin{array}{l}1.10(-1) \\
1.09 \\
1.08 \\
1.04 \\
9.70(-2)\end{array}$ & $\begin{array}{l}2.19(-1) \\
2.17 \\
2.14 \\
2.06 \\
1.93\end{array}$ \\
\hline 0.2 & $\begin{array}{r}6.26 \\
* 6.28\end{array}$ & $\begin{array}{r}\text { 1. } 24 \\
\text { *1. } 24\end{array}$ & $\begin{array}{r}2.93 \\
*_{2} .93\end{array}$ & $\begin{array}{r}4.99 \\
* 5.00\end{array}$ & $\begin{array}{r}8.53 \\
* 8.53\end{array}$ & $\begin{array}{r}1.69 \\
* 1.69\end{array}$ \\
\hline 0.5 & $\begin{array}{r}4.47 \\
* 4.45\end{array}$ & $\begin{array}{l}8.85(-2) \\
* 8.93\end{array}$ & $\begin{array}{r}2.07 \\
* 2.07\end{array}$ & $\begin{array}{r}3.45 \\
* 3.46\end{array}$ & $\begin{array}{r}5.82 \\
* 5.79\end{array}$ & $\begin{array}{l}1.15 \\
* 1.15\end{array}$ \\
\hline 1. 0 & $\begin{array}{r}2.56 \\
* 2.57\end{array}$ & $\begin{array}{r}5.06 \\
* 5.06\end{array}$ & $\begin{array}{r}1.17 \\
* 1.17\end{array}$ & $\begin{array}{r}1.87 \\
* 1.87\end{array}$ & $\begin{array}{r}3.14 \\
* 3.14\end{array}$ & $\begin{array}{r}6.20(-2) \\
* 6.21\end{array}$ \\
\hline 2.0 & $\begin{array}{r}8.49(-3) \\
* 8.53\end{array}$ & $\begin{array}{r}1.67 \\
* 1.67\end{array}$ & $\begin{array}{r}3.74 \\
* 3.74\end{array}$ & $\begin{array}{r}5.61(-2) \\
* 5.62\end{array}$ & $\begin{array}{r}9.57(-3) \\
* 9.60\end{array}$ & $\begin{array}{r}1.88 \\
*_{1} .88\end{array}$ \\
\hline $\begin{array}{r}5.0 \\
10.0\end{array}$ & $\begin{array}{l}3.85(-4) \\
* 3.29 \\
* 1.67 \quad(-6)\end{array}$ & $\begin{array}{l}7.55(-4) \\
* 6.35 \\
* 3.16(-6)\end{array}$ & $\begin{array}{r}1.77(-3) \\
{ }^{*} 1.31 \\
-*_{5} .77(-6)\end{array}$ & $\begin{array}{r}7.99(-3) \\
* 1.69 \\
* 6.49(-6)\end{array}$ & $\begin{array}{l}{ }^{*} 3.37(-4) \\
{ }^{*} 1.67(-6)\end{array}$ & $\begin{array}{l}* 6.54 \quad(-4) \\
* 3.16 \quad(-6)\end{array}$ \\
\hline
\end{tabular}


TABLE 4. Unscattered flux $(4 \pi / \sigma) \mathrm{I}^{\circ}(\tau, a, b)$ of radiation, at a corner position, from a rectangular plane isotropic source calculated using tables 1 and 2 (numbers preceded by asterisks) and using table 3 (no asterisks) - Con.

\begin{tabular}{|c|c|c|c|c|c|c|}
\hline$\tau$ & $\begin{array}{l}b=2.0 \\
a=0.5\end{array}$ & $\begin{array}{l}b=2.0 \\
a=1.0\end{array}$ & $\begin{array}{l}b=2.0 \\
a=2.0\end{array}$ & $\begin{array}{l}b=5.0 \\
a=0.1\end{array}$ & $\begin{array}{l}b=5.0 \\
a=0.2\end{array}$ & $\begin{array}{l}b=5.0 \\
a=0.5\end{array}$ \\
\hline $\begin{array}{l}0 \\
0.01 \\
0.02 \\
0.05 \\
0.1\end{array}$ & $\begin{array}{l}\text { 5. } 25(-1) \\
5.18 \\
5.11 \\
4.91 \\
4.60\end{array}$ & $\begin{array}{l}\text { 9. } 31(-1) \\
9.18 \\
9.05 \\
8.67 \\
8.07\end{array}$ & $\begin{array}{l}1.41(0) \\
1.38 \\
1.36 \\
1.30 \\
1.20\end{array}$ & $\begin{array}{l}\text { 1. } 37(-1) \\
\text { 1. } 35 \\
1.33 \\
1.26 \\
1.16\end{array}$ & $\begin{array}{l}2.73(-1) \\
2.68 \\
2.64 \\
2.51 \\
2.31\end{array}$ & $\begin{array}{l}6.57(-1) \\
6.46 \\
6.35 \\
6.03 \\
5.55\end{array}$ \\
\hline 0.2 & $\begin{array}{r}4.03 \\
* 4.02\end{array}$ & $\begin{array}{l}7.01 \\
* 6.99\end{array}$ & $\begin{array}{r}1.02 \\
{ }_{*} 1.02\end{array}$ & $\begin{array}{r}9.93 \\
* 9.97\end{array}$ & $\begin{array}{l}1.97 \\
* 1.98\end{array}$ & $\begin{array}{r}4.72 \\
* 4.73\end{array}$ \\
\hline 0.5 & $\begin{array}{r}\text { 2. } 72 \\
*_{2} .72\end{array}$ & $\begin{array}{r}4.62 \\
*_{4} .62\end{array}$ & $\begin{array}{r}6.38(-1) \\
* 6.39\end{array}$ & $\begin{array}{r}6.38 \\
* 6.35\end{array}$ & $\begin{array}{r}\text { 1. } 27 \\
{ }^{*} 1.26\end{array}$ & $\begin{array}{r}3.00 \\
* 2.99\end{array}$ \\
\hline 1.0 & $\begin{array}{r}1.44 \\
{ }^{*} 1.44\end{array}$ & $\begin{array}{l}2.35 \\
*_{2} .35\end{array}$ & $\begin{array}{r}3.02 \\
* 3.02\end{array}$ & $\begin{array}{r}\text { 3. } 27 \\
\text { *3. } 27\end{array}$ & $\begin{array}{l}\text { 6. } 46(-2) \\
* 6.45\end{array}$ & $\begin{array}{l}1.50 \\
* 1.50\end{array}$ \\
\hline \multirow{3}{*}{$\begin{array}{r}2.0 \\
5.0 \\
10.0\end{array}$} & \multirow{3}{*}{ 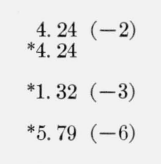 } & \multirow{3}{*}{$\begin{array}{l}6.43(-2) \\
* 6.43 \\
* 1.75(-3) \\
* 6.51(-6)\end{array}$} & \multirow{3}{*}{$\begin{array}{l}7.46(-2) \\
* 7.45 \\
* 1.80(-3) \\
* 6.52(-6) \\
\end{array}$} & \multirow{3}{*}{$\begin{array}{l}* 9.69(-3) \\
* 3.37(-4) \\
* 1.67 \quad(-6)\end{array}$} & \multirow{3}{*}{$\begin{array}{l}{ }^{*} 1.90 \\
* 6.54(-4) \\
* 3.16 \quad(-6)\end{array}$} & \multirow{3}{*}{$\begin{array}{ll}* 4.28 & (-2) \\
* 1.35 & (-3) \\
* & \\
* & \\
* & (-6)\end{array}$} \\
\hline & & & & & & \\
\hline & & & & & & \\
\hline$\tau$ & $\begin{array}{l}b=5.0 \\
a=1.0\end{array}$ & $\begin{array}{l}b=5.0 \\
a=2.0\end{array}$ & $\begin{array}{l}b=5.0 \\
a=5.0\end{array}$ & $\begin{array}{c}b=10.0 \\
a=0.1\end{array}$ & $\begin{array}{c}b=10.0 \\
a=0.2\end{array}$ & $\begin{array}{l}b=10.0 \\
a=0.5\end{array}$ \\
\hline $\begin{array}{l}0 \\
0.01 \\
0.02 \\
0.05 \\
0.1\end{array}$ & $\begin{array}{l}1.19 \quad(0) \\
1.17 \\
1.15 \\
1.08 \\
9.92 \quad(-1)\end{array}$ & $\begin{array}{ll}1.88 \quad(0) \\
1.84 \\
1.80 \\
1.69 \\
1.53\end{array}$ & $\begin{array}{ll}2.73 & (0) \\
2.65 & \\
2.58 & \\
2.39 & \\
2.10 & \end{array}$ & $\begin{array}{l}1.47(-1) \\
1.44 \\
1.41 \\
1.33 \\
1.21\end{array}$ & $\begin{array}{l}2.92(-1) \\
2.86 \\
2.81 \\
2.65 \\
2.41\end{array}$ & $\begin{array}{l}7.06(-1) \\
6.92 \\
6.77 \\
6.38 \\
5.79\end{array}$ \\
\hline 0.2 & $\begin{array}{r}8.35 \\
* 8.37\end{array}$ & $\begin{array}{r}1.26 \\
* 1.26\end{array}$ & $\begin{array}{l}1.64 \\
*_{1} .65\end{array}$ & $\begin{array}{l}1.02 \\
*_{1.02}\end{array}$ & $\begin{array}{l}2.02 \\
*_{2.02}\end{array}$ & $\begin{array}{r}4.84 \\
* 4.84\end{array}$ \\
\hline 0.5 & $\begin{array}{r}5.14 \\
* 5.14\end{array}$ & $\begin{array}{r}7.27 \\
* 7.27\end{array}$ & $\begin{array}{r}8.53(-1) \\
* 8.49\end{array}$ & $\begin{array}{r}6.42 \\
* 6.39\end{array}$ & $\begin{array}{r}1.27 \\
{ }^{*} 1.27\end{array}$ & $\begin{array}{r}\text { 3. } 01 \\
* 3.01\end{array}$ \\
\hline \multirow{4}{*}{$\begin{array}{r}1.0 \\
2.0 \\
5.0 \\
10.0\end{array}$} & \multirow{4}{*}{ 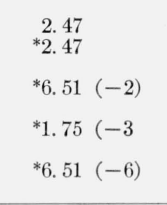 } & \multirow{4}{*}{ 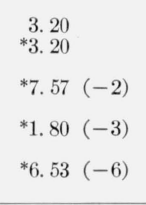 } & \multirow{4}{*}{ 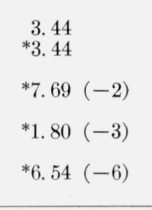 } & \multirow{4}{*}{$\begin{array}{l}\text { *3. } 27 \\
\text { *9. } 69(-3) \\
* 3.37(-4) \\
\text { *1. } 67 \quad(-6)\end{array}$} & \multirow{4}{*}{$\begin{array}{l}{ }^{*} 6.47(-2) \\
{ }^{*} 1.90 \\
{ }^{*} 6.54(-4) \\
* 3.16(-6)\end{array}$} & \multirow{4}{*}{$\begin{array}{l}{ }^{*} 1.50 \\
{ }^{*} 4.28(-2) \\
{ }^{*} 1.35(-3) \\
{ }^{*} 5.79(-6)\end{array}$} \\
\hline & & & & & & \\
\hline & & & & & & \\
\hline & & & & & & \\
\hline$\tau$ & $\begin{array}{c}b=10.0 \\
a=1.0\end{array}$ & $\begin{array}{c}b=10.0 \\
a=2.0\end{array}$ & $\begin{array}{c}b=10.0 \\
a=5.0\end{array}$ & $\begin{array}{l}b=10.0 \\
a=10.0\end{array}$ & $\begin{array}{c}b=20.0 \\
a=0.1\end{array}$ & $\begin{array}{c}b=20.0 \\
a=0.2\end{array}$ \\
\hline $\begin{array}{l}0 \\
0.01 \\
0.02 \\
0.05 \\
0.1\end{array}$ & $\begin{array}{ll}1.28 & (0) \\
1.26 & \\
1.23 & \\
1.15 & \\
1.04 & \end{array}$ & $\begin{array}{ll}2.07 & (0) \\
2.02 & \\
1.97 & \\
1.83 & \\
1.63 & \end{array}$ & $\begin{array}{l}3.15 \quad(0) \\
3.04 \\
2.94 \\
2.67 \\
2.30\end{array}$ & $\begin{array}{ll}3.80 \quad(0) \\
3.64 \\
3.49 \\
3.10 \\
2.58\end{array}$ & $\begin{array}{l}\text { 1. } 52(-1) \\
1.48 \\
1.45 \\
1.36 \\
1.22\end{array}$ & $\begin{array}{l}3.02(-1) \\
2.95 \\
2.88 \\
2.70 \\
2.43\end{array}$ \\
\hline 0.2 & $\begin{array}{l}\text { 8. } 60 \\
* 8.58\end{array}(-1)$ & $\begin{array}{l}\text { 1. } 31 \\
* 1.30\end{array}$ & $\begin{array}{l}1.74 \\
* 1.73\end{array}$ & $\begin{array}{l}1.86 \\
* 1.83\end{array}$ & $\begin{array}{l}1.02 \\
* 1.06\end{array}$ & $\begin{array}{l}2.03 \\
* 2.03\end{array}$ \\
\hline \multirow{2}{*}{$\begin{array}{l}0.5 \\
1.0\end{array}$} & $\begin{array}{r}5.18 \\
* 5.18\end{array}$ & \multirow{4}{*}{ 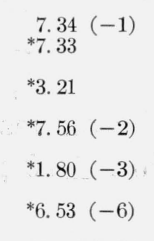 } & \multirow{4}{*}{$\begin{array}{l}8.65(-1) \\
{ }^{*} .62 \\
* 3.44 \\
* 7.69(-2) \\
{ }^{*} 1.80(-3) \\
* 6.53(-6)\end{array}$} & \multirow{4}{*}{ 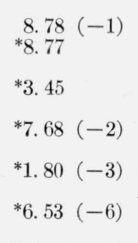 } & \multirow{4}{*}{$\begin{array}{l}* 6.39(-2) \\
* 3.27 \\
* 9.69(-3) \\
* 3.37(-4) \\
* 1.67(-6)\end{array}$} & \multirow{4}{*}{$\begin{array}{l}{ }^{*} 1.27 \\
* 6.47 \quad(-2) \\
{ }^{*} 1.90 \\
* 6.54(-4) \\
* 3.16(-6)\end{array}$} \\
\hline & ${ }^{*} 2.47$ & & & & & \\
\hline 5.0 & $* 6.51 \quad(-2)$ & & & & & \\
\hline $\begin{array}{r}5.0 \\
10.0\end{array}$ & $\begin{array}{l}{ }^{*} 1.75(-3) \\
{ }^{*} 6.51(-6)\end{array}$ & & & & & \\
\hline$\tau$ & $\begin{aligned} b & =20.0 \\
a & =0.5\end{aligned}$ & $\begin{array}{c}b=20.0 \\
a=1.0\end{array}$ & $\begin{array}{c}b=20.0 \\
a=2.0\end{array}$ & $\begin{array}{c}b=20.0 \\
a=5.0\end{array}$ & $\begin{array}{l}b=20.0 \\
a=10.0\end{array}$ & $\begin{array}{l}b=20.0 \\
a=20.0\end{array}$ \\
\hline $\begin{array}{l}0 \\
0.01 \\
0.02 \\
0.05 \\
0.1\end{array}$ & $\begin{array}{l}7.31(-1) \\
7.13 \\
6.96 \\
6.50 \\
5.86\end{array}$ & $\begin{array}{ll}1.33 & (0) \\
1.30 & \\
1.27 & \\
1.18 & \\
1.05 & \end{array}$ & $\begin{array}{ll}2.17 & (0) \\
2.10 & \\
2.04 & \\
1.88 & \\
1.65 & \end{array}$ & $\begin{array}{ll}3.38 & (0) \\
3.25 & \\
3.12 & \\
2.79 & \\
2.36 & \end{array}$ & $\begin{array}{ll}\text { 4. } 22 & (0) \\
\text { 4. } 01 & \\
3.81 & \\
3.30 & \\
2.68 & \end{array}$ & $\begin{array}{ll}4.88 & (0) \\
4.56 & \\
4.28 & \\
3.59 & \\
2.81 & \end{array}$ \\
\hline 0.2 & $\begin{array}{r}4.86 \\
* 4.85\end{array}$ & $\begin{array}{r}8.63(-1) \\
\text { *.62 }\end{array}$ & $\begin{array}{r}1.31 \\
*_{1} .31\end{array}$ & $\begin{array}{l}1.75 \\
* 1.74\end{array}$ & $\begin{array}{l}1.89 \\
* 1.86\end{array}$ & $\begin{array}{r}1.92 \\
* 1.89\end{array}$ \\
\hline $\begin{array}{l}0.5 \\
1.0\end{array}$ & $* 3.01$ & ${ }^{*} 5.18$ & $* 7.34$ & *8. $63(-1)$ & *8. $79(-1)$ & *8. $82(-1)$ \\
\hline $\begin{array}{l}1.0 \\
2.0\end{array}$ & ${ }^{*} 1.50$ & $*_{2.47}$ & ${ }^{*} 3.21$ & $* 3.44$ & $* 3.45$ & $* 3.45$ \\
\hline 5.0 & *4. $28(-2)$ & $* 6.51 \quad(-2)$ & $* 7.56(-2)$ & $* 7.69 \quad(-2)$ & *7. $68(-2)$ & $* 7.68(-2)$ \\
\hline 10.0 & ${ }^{*} 1.35 \quad(-3)$ & $* 1.75 \quad(-3)$ & $* 1.80 \quad(-3)$ & ${ }^{*} 1.80 \quad(-3)$ & $* 1.80 \quad(-3)$ & $* 1.80 \quad(-3)$ \\
\hline & *5.79 (-6) & $* 6.51 \quad(-6)$ & $* 6.53(-6)$ & ${ }^{*} 6.53(-6)$ & *6. $53(-6)$ & ${ }^{*} 6.53(-6)$ \\
\hline
\end{tabular}




\section{Examples}

Comparison of the results in the above sections 4 and 5 is made in table 5 with similar results of Berger and Lamkin [1] for a water barrier of $\tau=4$ and $a=b=2$, using the compounding rule in section 3 for noncorner positions. Agreement is within 5 percent.

There exists little experimental information on flux fields from rectangular gamma-ray sources. However, an additional partial check on $q_{0}(a, b)$ was supplied by Auxier et al., [3] as shown by the circles, triangles, and squares in figure 7 . The distributed source was represented by an array of matched $( \pm 5 \%) \mathrm{Co}^{60}$ sources spaced $2 \mathrm{ft}$ apart over a 24 - by 32 -ft rectangle. The detectors were pocket ionization chambers with an average response of 14.4 milliroentgens per hour at $1 \mathrm{ft}$ from a 1 -millicurie point $\mathrm{Co}^{60}$ source. This response calibration was made in a geometry such that the combined effects of backscattering from the ground, the buildup factor in air, and the exponential attenuation by air were roughly equivalent to

TABLE 5. Dose rate, in milliroentgens per hour per millicuries per square foot of source strength, at a number of positions $\alpha=\mathrm{x} / \mathrm{h}, \beta=\mathrm{y} / \mathrm{h}$ at distance $\mathrm{h}$ from a rectangular surface of relative dimensions $\mathrm{w} / \mathrm{h}=2,1 / \mathrm{h}=2$ separated from a plane source of 1-Mev photons by a layer of water of thickness $\tau=4$

The numbers in parenthesis* are from table 1 of Berger and Lamkin [1].

\begin{tabular}{|c|c|c|c|c|c|}
\hline & 0 & 0.25 & 0.50 & 0.75 & 1.0 \\
\hline 0 & $\begin{array}{l}0.211 \\
(.22)\end{array}$ & & & & \\
\hline .25 & $\begin{array}{l}.275 \\
(.28)\end{array}$ & $\begin{array}{c}0.361 \\
(.37)\end{array}$ & & & \\
\hline .50 & $\begin{array}{l}.323 \\
(.33)\end{array}$ & $\begin{array}{l}425 \\
(.44)\end{array}$ & $\begin{array}{c}0.503 \\
(.51)\end{array}$ & & \\
\hline .75 & $\begin{array}{l}.350 \\
(.35)\end{array}$ & $\begin{array}{r}462 \\
(.48)\end{array}$ & $\begin{array}{l}.546 \\
(.55)\end{array}$ & $\begin{array}{c}0.593 \\
(.60)\end{array}$ & \\
\hline 1. 0 & $\begin{array}{l}.359 \\
(.37)\end{array}$ & $\begin{array}{l}.473 \\
(.49)\end{array}$ & $\begin{array}{l}.559 \\
(.57)\end{array}$ & $\begin{array}{l}.608 \\
(.62)\end{array}$ & $\begin{array}{c}0.624 \\
(.63)\end{array}$ \\
\hline
\end{tabular}

${ }^{*}$ Arithmetic error in the Berger-Lamkin result at $\alpha=0.25, \beta=0.25$ is here corrected.

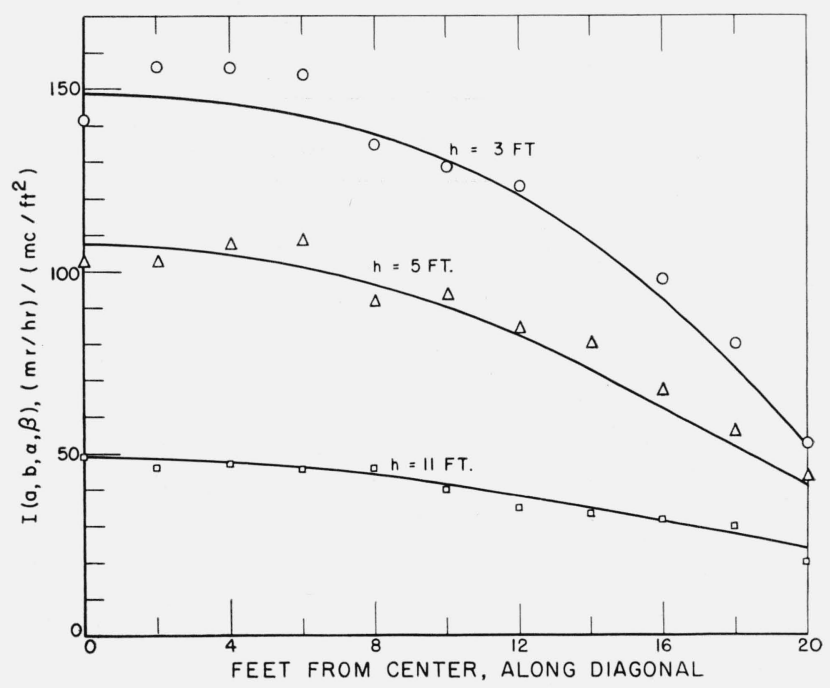

FIGURE 7. Detector response in milliroentgens per hour at $h=3$, 5 and $11 \mathrm{ft}$ above the diagonal of a rectangle of dimensions $w=24 \mathrm{ft}, \quad l=32 \mathrm{ft}$ covered with one millicurie per foot [2] of $\mathrm{Co}^{60}$. The circles, triangles, and squares are experimental values of Auxier et al. [3] and the curves are results from (28) compounded according to (11).

these combined effects in the actual measurements. Thus, since the experimental data in figure 7 are related to a source strength of 1 millicurie per square foot, the dimensional factor $\sigma / 4 \pi$ in (10) may be identified as 14.4 milliroentgens per square foot, and we may multiply $q_{0}(a, b ; \alpha, \beta)$ by this number for an absolute comparison. The curves in figure 7 represent $14.4 \cdot q(a, b ; \alpha, \beta)$, and agree with the experimental data to within \pm 10 percent despite neglect of attenuation and scattering effects.

The authors thank M. J. Berger and L. V. Spencer for their interest, encouragement, and helpful criticism regarding this effort.

\section{Appendix A. Evaluation of the $\mathrm{p}_{l}(\mathrm{a}, \mathrm{b})$ Coefficients}

The Legendre polynomials, $P_{l}(\cos \theta)$, may be written in the form [13]

$$
P_{l}(\cos \theta)=\sum_{j=0}^{m} \frac{(-1)^{j}(2 l-2 j) !}{2^{l} j !(l-j) !(l-2 j) !}(\cos \theta)^{l-2 j},
$$

where $m=l / 2$ if $l$ is even, and $m=(l-1) / 2$ if $l$ is odd. Substituting (34) into (15) and using the notation 


$$
\left(\begin{array}{l}
n \\
r
\end{array}\right)=\frac{n !}{(n-r) ! r !}
$$

for the $r$ 'th coefficient of an $n$ 'th degree binomial expansion, we have for the even $p_{l}$ 's

$$
p_{2 l}=\sum_{j=0}^{l} \frac{(-1)^{j}}{2^{2 l}}\left(\begin{array}{c}
2 l-j \\
j
\end{array}\right)\left(\begin{array}{c}
4 l-2 j \\
2 l-j
\end{array}\right) \int_{\alpha=0}^{a} \int_{\beta=0}^{b} \frac{d \alpha d \beta}{\left(\alpha^{2}+\beta^{2}+1\right)^{l-j+3 / 2}} .
$$

Integration over $\alpha$ yields[14]

$$
p_{2 l}=\sum_{j=0}^{l} \sum_{i=0}^{l-j} \frac{(-1)^{i+j}}{2^{2 l}(2 i+1)}\left(\begin{array}{c}
2 l-j \\
j
\end{array}\right)\left(\begin{array}{c}
4 l-2 j \\
2 l-j
\end{array}\right)\left(\begin{array}{c}
l-j \\
i
\end{array}\right) a^{2 i+1} \int_{0}^{b} \frac{d \beta}{\left(\beta^{2}+1\right)^{l}-{ }^{j+1}\left(a^{2}+\beta^{2}+1\right)^{i+\frac{1}{2}}} .
$$

For $l=0$, integration over $\beta$, using eq $(234.19 \mathrm{a}$, reference [14], yields the result in (16). For the higher even terms, use of eq (234.18a) leads to (18) and higher terms which may be expressed in the general form

$$
\begin{aligned}
p_{2 l}= & \frac{1}{l(2 l+1) 2^{2 l}} \frac{a b}{\sqrt{a^{2}+b^{2}+1}} \sum_{k=0}^{l-1} \frac{(-1)^{i-k-1} 2^{2 k}\left(\begin{array}{c}
2 l+2 k \\
l+k
\end{array}\right)\left(\begin{array}{c}
l+k \\
l-k-1
\end{array}\right)}{\left(\begin{array}{c}
2 k \\
k
\end{array}\right)(2 k+1)} \\
& \times(2 l+2 k+1) \sum_{j=0}^{k} \frac{\left(\begin{array}{c}
2 j \\
j
\end{array}\right)}{2^{2 j}} \frac{1}{\left(a^{2}+b^{2}+1\right)^{j}}\left(\frac{1}{\left(a^{2}+1\right)^{k-j+1}}+\frac{1}{\left(b^{2}+1\right)^{k-j+1}}\right) .
\end{aligned}
$$

The odd $p_{l}$ 's as obtained from (15) and (34) may be expressed as

$$
p_{2 l+1}=\sum_{j=0}^{l} \frac{(-1)^{j}}{2^{2 l+1}}\left(\begin{array}{c}
2 l+1-j \\
j
\end{array}\right)\left(\begin{array}{c}
4 l+2-2 j \\
2 l+1-j
\end{array}\right) \int_{\alpha=0}^{a} \int_{\beta=0}^{b} \frac{d \alpha d \beta}{\left(\alpha^{2}+\beta^{2}+1\right)^{l-j+2}} .
$$

Integration over $\alpha$ yields (see ref. 14 eqs $15,15 \mathrm{a}, \mathrm{b}$ )

$$
\begin{aligned}
p_{2 l+1}= & \frac{1}{2^{2 l+2}} \sum_{j=0}^{l}(-1)^{j}\left(\begin{array}{c}
2 l+1-j \\
j
\end{array}\right)\left(\begin{array}{c}
4 l+2-2 j \\
2 l+1-j
\end{array}\right)\left(\begin{array}{c}
2 l-2 j+1 \\
l-j
\end{array}\right) \\
& \times\left[\frac{1}{2^{3 l-3 j}} \int_{\beta=0}^{b} \frac{\tan ^{-1}\left(a / \sqrt{\beta^{2}+1}\right) d \beta}{\left(\beta^{2}+1\right)^{l-j+3 / 2}}\right. \\
& \left.+a \sum_{i=0}^{l-j} \frac{1}{2^{2 i}\left(\begin{array}{c}
2 l-2 j-2 i+1 \\
l-j-i
\end{array}\right)(l-j-i+1)} \int_{\beta=0}^{b} \frac{d \beta}{\left(\beta^{2}+1\right)^{i+1}\left(a^{2}+\beta^{2}+1\right)^{l-j-i+1}}\right] .
\end{aligned}
$$

The integral containing the arctangent was amenable to integration by parts, and the purely algebraic integrand was reduced to terms of standard form by successive application of the identity

$$
\frac{1}{\left(\beta^{2}+1\right)\left(a^{2}+\beta^{2}+1\right)}=\frac{1}{a^{2}}\left(\frac{1}{\beta^{2}+1}-\frac{1}{a^{2}+\beta^{2}+1}\right),
$$

leading to the general form for the odd terms, including (17) and (19),

$$
\begin{aligned}
p_{2 l+1}=\frac{1}{2^{2 l+1}(2 l+1)} & \sum_{k=0}^{l} \frac{(-1)^{l+k}}{2^{2 k}}\left(\begin{array}{l}
l \\
k
\end{array}\right)\left(\begin{array}{c}
l+k+1 \\
k
\end{array}\right)\left(\begin{array}{c}
2 l+2 k+1 \\
l+k
\end{array}\right) \\
\times & \left\{\frac{1}{\left(b^{2}+1\right)^{k}} \frac{b}{\sqrt{b^{2}+1}} \tan ^{-1} \frac{a}{\sqrt{b^{2}+1}}+\frac{1}{\left(a^{2}+1\right)^{k}} \frac{a}{\sqrt{a^{2}+1}} \tan ^{-1} \frac{b}{\sqrt{a^{2}+1}}\right. \\
& \left.+a b \sum_{j=1}^{k} \frac{2^{2 j-1}}{j\left({ }^{2 j}\right)} \cdot \frac{1}{\left(a^{2}+b^{2}+1\right)^{j}}\left(\frac{1}{\left(a^{2}+1\right)^{k-j+1}}+\frac{1}{\left(b^{2}+1\right)^{k-j+1}}\right)\right\} .
\end{aligned}
$$




\section{Appendix B. Evaluation of the $q_{n}(a, b)$ Coefficients}

The integral (27) may be written in cartesian coordinates as

The substitution

$$
I^{o}(\tau, a, b)=\frac{\sigma}{4 \pi} \int_{0}^{a} d \alpha \int_{0}^{b} d \beta \exp \left(-\tau \sqrt{\alpha^{2}+\beta^{2}+1}\right) /\left(\alpha^{2}+\beta^{2}+1\right) .
$$

yields

$$
\alpha=\sqrt{\beta^{2}+1} \tan \eta
$$

$$
I^{o}(\tau, a, b)=\frac{\sigma}{4 \pi} \int_{0}^{b} \frac{d \beta}{\sqrt{\beta^{2}+1}} \int_{0}^{\tan ^{-1} \frac{a}{\sqrt{\beta^{2}+1}}} \exp \left(-\tau \sqrt{\beta^{2}+1} \sec \eta\right) d \eta .
$$

Expanding the exponential as a power series of its argument we have

$$
I^{o}(\tau, a, b)=\frac{\sigma}{4 \pi} \int_{0}^{b} \frac{d \beta}{\sqrt{\beta^{2}+1}} \int_{0}^{\tan ^{-1} \frac{a}{\sqrt{\beta^{2}+1}}}\left\{\sum_{n=0}^{\infty} \frac{1}{n !}\left(-\tau \sqrt{\beta^{2}+1} \text { sec } \eta\right)^{n}\right\} d \eta .
$$

The first integration is then simply over powers of sec $\eta$, which can be found in integral tables as standard forms [15], giving

$$
\begin{aligned}
I^{o}(\tau, a, b) & =\frac{\sigma}{4 \pi} \int_{0}^{b}\left\{\frac{1}{\sqrt{\beta^{2}+1}} \tan ^{-1} \frac{a}{\sqrt{\beta^{2}+1}}-\tau \sinh ^{-1} \frac{a}{\sqrt{\beta^{2}+1}}\right. \\
& \left.+\frac{\tau^{2}}{2 !} a-\frac{\tau^{3}}{3 !}\left[a \frac{\sqrt{a^{2}+\beta+1}}{2}+\frac{\sqrt{\beta^{2}+1}}{2} \sinh ^{-1} \frac{a}{\sqrt{\beta^{2}+1}}\right]+\frac{\tau^{4}}{4 !}\left[\frac{a^{3}}{3}+a\left(\beta^{2}+1\right)\right]-\ldots\right\} d \beta .
\end{aligned}
$$

Integration term by term over $\beta$ then yields a power series in $\tau$ of the desired form (10), in which the coefficients $q_{n}(a, b)$ are

$$
\begin{aligned}
& q_{0}(a, b)=\int_{0}^{b} \frac{1}{\sqrt{\beta^{2}+1}} \tan ^{-1} \frac{a}{\sqrt{\beta^{2}+1}} d \beta, \\
& q_{1}(a, b)=-\int_{0}^{b} \sinh ^{-1} \frac{a}{\sqrt{\beta^{2}+1}} d \beta, \\
& q_{2}(a, b)=\frac{a}{2 !} \int_{0}^{b} d \beta, \text { etc. }
\end{aligned}
$$

as evaluated using (47).

No success was had in evaluating $q_{0}(a, b)$ in closed form. However, use of the arctangent expansion [16]

$$
x \tan ^{-1} x=\sum_{m=0}^{\infty} \frac{2^{2 m}(m !)^{2}}{(2 m+1) !}\left(\frac{x^{2}}{1+x^{2}}\right)^{m+1}
$$

yields the series of integrable terms

$$
q_{0}(a, b)=\frac{1}{a} \int_{0}^{b} \sum_{m=1}^{\infty} \frac{2^{2 m}(m !)^{2}}{(2 m+1) !}\left(\frac{a^{2}}{a^{2}+\beta^{2}+1}\right)^{m+1} d \beta .
$$

Integration of these terms (see ref 15, p. 25) gives the result

$$
\begin{aligned}
q_{0}(a, b)=\tan ^{-1} \frac{b}{\sqrt{a^{2}+1}} \cdot \frac{\sqrt{a^{2}+1}}{a} \sum_{i=0}^{\infty} \frac{1}{2 i+1}\left(\frac{a^{2}}{a^{2}+1}\right)^{i+1} & \\
& \quad+\frac{b}{a} \sum_{i=0}^{\infty} \frac{1}{2 i+1}\left(\frac{a^{2}}{a^{2}+1}\right)^{i+1} \sum_{j=0}^{i-1} \frac{2^{2 j}(j !)^{2}}{(2 j+1) !}\left(\frac{a^{2}+1}{a^{2}+b^{2}+1}\right)^{j+1} .
\end{aligned}
$$


in which the double summation vanishes for $i=0$. Since convergence of the summations in (53) is poor when both $a$ and $b$ are large as compared with unity, it is advantageous to rewrite the first member of the right-hand side, using the substitutions

$$
\tan ^{-1} \frac{b}{\sqrt{a^{2}+1}}=\frac{\pi}{2}-\tan ^{-1} \frac{\sqrt{a^{2}+1}}{b}
$$

and

$$
\frac{\sqrt{a^{2}+1}}{a} \sum_{i=0}^{\infty} \frac{1}{2 i+1}\left(\frac{a^{2}}{a^{2}+1}\right)^{i+1}=\tanh ^{-1} \frac{a}{\sqrt{a^{2}+1}}=\sinh ^{-1} a .
$$

This gives

$$
\begin{aligned}
q_{0}(a, b)=\frac{\pi}{2} \sinh ^{-1} a-\tan ^{-1} \frac{\sqrt{a^{2}+1}}{b} \cdot \frac{\sqrt{a^{2}+1}}{a} \sum_{i=0}^{\infty} \frac{1}{2 i+1}\left(\frac{a^{2}}{a^{2}+1}\right)^{i+1} & \\
& +\frac{b}{a} \sum_{i=0}^{\infty} \frac{1}{2 i+1}\left(\frac{a^{2}}{a^{2}+1}\right)^{i+1} \sum_{j=0}^{i-1} \frac{2^{2 j}(j !)^{2}}{(2 j+1) !}\left(\frac{a^{2}+1}{a^{2}+b^{2}+1}\right)^{j+1}
\end{aligned}
$$

which, after factoring the quantity

$$
-\frac{b}{a} \sum_{i=0}^{\infty} \frac{1}{2 i+1}\left(\frac{a^{2}}{a^{2}+1}\right)^{i+1}
$$

from the last two members, may be identified with the result in eq (28). Now, since the inner summation over $j$ in (28) consists of the leading terms of the expansion of $\left(\sqrt{a^{2}+1} / b\right)$ $\times \tan ^{-1}\left(\sqrt{a^{2}+1} / b\right)$ according to (51), the quantity within the braces in (28) is the remainder after $i-1$ terms of this expansion, causing the outer summation over $i$ to converge very quickly for all values of $a$ and $b$.

The remaining $q_{n}(a, b)$ coefficients for $n$ even and odd, respectively, may be written in the general forms

$$
q_{2 n}(a, b)=\frac{1}{(2 n) !}\left\{(-1)^{n} \sum_{i=0}^{n-2}(-1)^{i}\left(\begin{array}{c}
n-1 \\
i
\end{array}\right)(2 i+2) ! q_{2 i+2}(a, b)+\sum_{i=0}^{n-1}\left(\begin{array}{c}
n-1 \\
i
\end{array}\right) \frac{a^{2 n-2 i-1} b^{2 i+1}}{(2 n-2 i-1)(2 i+1)}\right\}
$$

and

$$
\begin{aligned}
q_{2 n+1}(a, b) & =-\frac{1}{(2 n+1) !}\left\{(2 n-1) ! \frac{2 n-1}{2 n+1}\left[-q_{2 n-1}(a, b)\right]\right. \\
& +\frac{2 n !}{2^{2 n}(n !)^{2}(2 n+1)}\left[b\left(b^{2}+1\right)^{n} \sinh ^{-1} \frac{a}{\sqrt{b^{2}+1}}+a\left(a^{2}+1\right)^{n} \sinh ^{-1} \frac{b}{\sqrt{a^{2}+1}}\right. \\
& \left.\left.+a b \sqrt{a^{2}+b^{2}+1} \sum_{i=0}^{n-1} \frac{2^{2 i}(i !)^{2}}{(2 i+1) !}\left(a^{2}+b^{2}+1\right)^{i}\left\{\left(a^{2}+1\right)^{n-i-1}+\left(b^{2}+1\right)^{n-i-1}\right\}\right]\right\}
\end{aligned}
$$

from which $q_{n}(a, b)$ 's for $1 \leq n \leq 4$ are given in (29 to 32 ).

\section{References}

[1] M. J. Berger and J. C. Lamkin, Sample calculations of gamma-ray penetration into shelters: contributions of sky shine and roof contamination, J. Research NBS 60, 109 (1958) RP2827.

[2] L. V. Spencer, Structure shielding against fallout radiation from nuclear weapons (to be published as an NBS Monograph).

[3] J. A. Auxier, J. O. Buchanan, C. Eisenhauer, and H. E. Menker, Experimental evaluation of the radiation protection afforded by residential structures against distributed sources, CEX 58.1, ORNL (Jan. 19, 1959). 
[4] U. Fano, L. V., Spencer, and M. J. Berger, Penetration and diffusion of X-rays, Encyclopedia of Physics (Springer-Verlag, 38 II, 1959).

[5] R. A. Herman, A treatise on geometrical optics, p. 210 (Cambridge Univ. Press, London, 1900).

[6] Z. Yamanouti, Geometrical calculation of illumination due to light from luminous sources of simple forms. Researches Electrotech. Lab. (Tokyo) No. 148 (1924).

[7] P. Moon and D. E. Spencer, Light distribution from rectangular sources, J. Franklin Inst. 241, 195 (1946).

[8] A. I. Mahan and W. F. Malmborg, Radiation characteristics of semicircular, circular and rectangular surface sources, J. Optical Soc. Amer. 44, 644 (1954).

[9] O. Seiber, Die Wärmeaufnahme der bestrahlten Kesselheizfläche, Arch. Wärmwirtsch. 9, 180 (1928).

[10] See, for example, W. B. Boast, Illuminating engineering, 1st ed., p. 89 (McGraw-Hill Book Co., New York, N.Y., 1942).

[11] G. Placzek, The function $E_{n}(x)=\int_{1}^{\infty} e^{-x u} u^{-n} d u$, MT-1, NRC No. 1547, Chalk River, Ont. (1946).

[12] D. G. Andrews, Gamma ray shielding for engineers, U.K.A.E.A., Report Risley 5225 (Sept. 1954).

[13] R. V. Churchill, Fourier series and boundary value problems, 1st ed., p. 177 (McGraw-Hill Book Co., New York, N.Y., 1941).

[14] W. Gröbner and N. Hofreiter, Integraltafel I, Eqn. 234.17b. (Unbestimmte Integrale), (Springer-Verlag, 1957).

[15] H. B. Dwight, Tables of Integrals and Other Mathematical Data, 3d ed., p. 97 (McGraw-Hill Book Co., New York, N.Y., 1957).

[16] E. P. Adams and R. L. Hippisley, Smithsonian mathematical formulae and tables of elliptic functions, p. 122 (Smithsonian Institution, Washington, D.C., 1922).

Washington, D. C.

(Paper 64C2-32) 